\title{
Transient viscoelastic flows in planar contractions
}

\author{
M.F. Webster ${ }^{1}$, H.R.Tamaddon-Jahromi, M. Aboubacar \\ Department of Computer Science, Institute of Non-Newtonian Fluid Mechanics, \\ University of Wales, Singleton Park, Swansea SA2 8PP, U.K.
}

\begin{abstract}
This article considers transient flows for planar contractions and Oldroyd-B fluids, with increasing flow-rate boundary conditions. We employ a novel hybrid finite volume/element time-dependent algorithm. The hybrid scheme consists of a TaylorGalerkin finite element discretisation, and a cell-vertex fluctuation-distribution finite volume approach. These two approaches are coupled at each time-step to solve the parabolic/hyperbolic system of partial differential equations. The finite element section is applied to the mass and momentum conservation equations, whilst the hyperbolic constitutive equation is treated via finite volume discretisation. The application of this time-accurate scheme fluids reveals some novel features, in contrast to time-independent (constant flow-rate) driving boundary conditions. In particular, we highlight dynamic flow structure evolution on the field and in stress.
\end{abstract}

Key words: Transient flows; Boundary conditions; Hybrid finite volume; Contraction geometries; Viscoelasticity

1 Corresponding author. Tel.: +44-1792-295656; fax: +44-1792-295708.

E-mail address: m.f.webster@swansea.ac.uk 


\section{Introduction and governing equations}

In the last two decades, much effort has been devoted to devising stable numerical algorithms for steady-state viscoelastic flows, see Keunings [1], Baaijens [2] and Walters and Webster [3] for reviews. Here, the main objective has been to achieve steady-state solutions of the associated elliptic/hyperbolic system of governing equations, for ever increasing Weissenberg numbers levels, see "High-Weissenberg Number Problem (HWNP)", Keunings [1]. This problem is now more clearly understood and may be mitigated via constitutive equations other than the Oldroyd/Maxwell type. With the advent of such stable numerical algorithms, attention has shifted towards the development of timedependent numerical methods. Indeed, such transient tools are necessary, if one is to resolve complex phenomena such as flow instabilities that can occur during industrial processing, extrusion being a typical example $[4,5]$.

In the context of the 4:1 contraction benchmark flow, we may cite the works of Olsson [6], Wapperom et al. [7], Bishko et al. [8] and Mompean and Deville [9]. Olsson [6] studied the flow of a Giesekus fluid through a 4:1 contraction, with a slight rounding of the corner. Olsson reported the appearance of a lip-vortex, that grew continuously with time to form a large recirculation region at steady-state, extending from the salient-corner to the re-entrant corner. Wapperom et al. [7] found a similar steady-state vortex for a FENE-P model, albeit with some difference in time evolution. In this case, recirculation appeared first within the salient-corner and grew subsequently. Eventually, a separate lip-vortex emerged, and as it grew, the salient-corner vortex shrank away. Finally, the dominant lip-vortex engulfed the salient-corner vortex, to produce, a single large recirculation. In both above works $[6,7]$, the authors imposed a static velocity-profile at the inlet (steady-state, constant flow-rate), with quiescent initial field conditions in all variables. We infer that transient evolution from this impulsive shock, start-up scenario is barely physical, with a constant flow-rate, fixed inlet-to-outlet pressure-drop. The rationale is that entry/exit stations are sufficiently far from contraction zones so as not to influence the dynamic flow evolution there. We cast light on this issue below.

In contrast, Bishko et al. [8] investigated an alternative transient flow evolution for a pom-pom fluid through a 4:1 contraction. Here, though their initial field-state was that of rest, these authors appealed to a transient evolving inlet velocity-profile for a 1D-channel flow. The corresponding inlet-profiles evolved from an initial Newtonian-form to that of shear-thinning at steadystate. Again, the lip of the contraction was slightly-rounded to mitigate numerical difficulties. There were no outstanding features observed in streamlinestructure as time-evolution advanced towards a steady-state. That is, in common with many other authors (but with the exception of Olsson), an increase is observed in the salient-corner vortex-size, followed by a subsequent decrease. 
In particular, there was no dynamic appearance of a lip-vortex reported by Bishko et al., noting that the corner was rounded.

Using finite volume methodology, Mompean and Deville documented the transient evolution of streamlines for an Oldroyd-B fluid in a 4:1 planar abruptcontraction [9]. There, the sharpness of the corner was not altered, and the polymeric-solvent, viscosity-split ratios followed convention: $\mu_{1} / \mu=8 / 9$ and $\mu_{2} / \mu=1 / 9$, respectively. The authors employed rectangular staggered grids, with extra-stress components located at cell-centres. Again, boundary conditions were time-independent (constant flow-rate and profile), and simulations commenced from a rest-state for a pressure-gradient driven flow. For a Deborah number $(D e)$ of 0.3 and $R e=0.01$, the Oldroyd-B salient-corner vortex cell-size increased from zero to a maximum, before declining to a steady-state value of 0.19 units. Mompean and Deville concluded that the major difference between Newtonian and Oldroyd-B fluids lay in the transient development of the salient-corner vortex-size. Alternatively, under identical boundary conditions (constant flow-rate) and for the same mesh, the Newtonian vortex-size development followed a pattern of monotonic increase from zero to its steadystate value of 0.19 units. Note, the identical steady-state value for Newtonian and Oldroyd-B reflects the relatively low-level of fluid-elasticity under consideration $(D e=0.3)$.

In this paper and in contrast to the foregoing, we investigate the influence of controlled, dynamic boundary conditions on evolutionary flow-structure in a start-up 4:1 contraction flow, for Oldroyd-B model fluids. We begin by introducing the governing equations and describing the problem specification in section 2. Subsequently, we summarize the key aspects concerning the numerical algorithm in section 3, before discussing our results in section 4. Finally, we draw our conclusions in section 5 .

\section{Governing equations and problem specification}

We consider isothermal incompressible viscoelastic flows. In non-dimensional form, with extra-stress $\boldsymbol{\tau}$, fluid velocity $\boldsymbol{u}$ and hydrodynamic pressure $p$, the governing equations can be expressed as:

$$
\begin{gathered}
\nabla \cdot \boldsymbol{u}=0 \\
R e \frac{\partial \boldsymbol{u}}{\partial t}=-R e \boldsymbol{u} \cdot \nabla \boldsymbol{u}-\nabla p+\nabla \cdot\left(2 \frac{\mu_{2}}{\mu} \boldsymbol{d}+\boldsymbol{\tau}\right) \\
W e \frac{\partial \boldsymbol{\tau}}{\partial t}=-W e \boldsymbol{u} \cdot \nabla \boldsymbol{\tau}+2 \frac{\mu_{1}}{\mu} \boldsymbol{d}+W e\left(\boldsymbol{L} \cdot \boldsymbol{\tau}+\boldsymbol{\tau} \cdot \boldsymbol{L}^{T}\right)-f \boldsymbol{\tau}
\end{gathered}
$$


Here, via identity function $f$, we retain the Oldroyd-B constitutive equation for the polymeric stress-contribution $\tau$. This provides a constant shear viscosity model-fluid, with memory effects. The Eulerian rate-of-deformation tensor is given by $\boldsymbol{d}=\left(\boldsymbol{L}+\boldsymbol{L}^{T}\right) / 2$, where $\boldsymbol{L}^{T}=\nabla \boldsymbol{u}$ is the velocity gradient. The total viscosity $\mu$ is split into Newtonian solvent $\left(\mu_{2}\right)$ and polymeric $\left(\mu_{1}\right)$ contributions, such that $\mu=\mu_{1}+\mu_{2}$. In our current study, we have chosen the standard polymeric-solvent viscosity-split values, that is $\mu_{1} / \mu_{2}=8$. This corresponds to a polymeric-viscosity dominated (constant-viscosity) fluid. This choice is dictated by the vast amount of data available in the literature, that provides a sound basis for comparison. In order to model Boger fluids closely, we note it would be customary to consider solvent-viscosity dominated fluids, as illustrated recently by Aboubacar et al. [10]. Elsewhere, we have had recourse to shear-thinning alternative versions, with capped extensional viscosity, via Phan-Thien/Tanner models of both exponential and linear form $[11,12]$, in steady-state context. Restriction to the Oldroyd model provides a well-categorised class of transient flow problems.

Under the non-dimensionalisation convention, group numbers of Reynolds and Weissenberg are defined accordingly as:

$$
R e=\frac{\rho U L}{\mu}, \quad W e=\frac{\lambda U}{L}
$$

where $\rho, \lambda$ are the fluid density and relaxation time; $U$ (die-exit, averagevelocity) and $L$ (die-exit, half-width) are characteristic-velocity and lengthscales for the flow. By assuming creeping flow conditions, the momentum convection terms may be discarded, whilst retaining transient terms to provide the time-stepping facility, see Carew et al. [13].

First, we appeal to a model problem, that of a planar start-up channel Poiseuilleflow to demonstrate the accuracy of the proposed numerical method in tracking the transient flow evolution, developing from rest to establish steady-state conditions. The reader is referred to Tamaddon-Jahromi et al. [14] for further details on variants of the transient algorithm. There, we discuss algorithm optimisation and start-up flow results, from which Fig. 1a illustrates a 10x10 unstructured mesh. This is found sufficient to suit present purposes, reflecting proximity of numerical transients against analytical counterparts (accuracy confirmed by time-step reduction through one order). This channel-flow problem, combined with the Oldroyd-B equation, is equipped with an analytical solution provided by Waters and King [15]; see also Carew et al. [16] and $\mathrm{Xu}$ et al. [17]. The problem is equivalent to applying an impulsive, constant pressure-gradient to drive the flow. 
Subsequently, we apply the aforementioned time-accurate algorithm to the benchmark 4:1 contraction flow. As in Fig. 1b, we retain the finest mesh employed in our previous studies, where mesh refinement was considered extensively $[12,18]$. As in the channel-flow scenario, simulations are instigated from rest, for each Weissenberg number (fluid) considered. We contrast both scenarios of standard time-independent and the present time-dependent inflowsetting on velocity and stress [15]. The latter settings induce a realistic transient flow-evolution with dynamic growth of flow-rate, see Fig. 5. Creeping flow is assumed throughout to suppress inertial contributions, that in themselves would only complicate matters still further.

For both flow settings, velocity components are imposed at inlet and exit stations, whilst stress is imposed at inlet only. In addition, a pressure level is set at a boundary exit point, hence removing indeterminacy on ambient pressure. No-slip boundary conditions prevail at the walls. For the contraction flow, we solve for the half-domain problem, symmetry being enforced thereby at the centreline. Typical time steps are of order $0\left(10^{-3}\right)$. A steady-state relative (unitless) increment time-stepping termination criteria is adopted of $10^{-6}$. The latter is found to be sufficiently stringent and governs a relative solution temporal increment norm, within a least squares measure [19, 20].

\section{$3 \quad$ Hybrid finite element/finite volume scheme}

The numerical method employed in this study is discussed extensively elsewhere [14]. Hence, we provide only a brief overview here. The underlying finite element $(f e)$ framework is that of a time-splitting semi-implicit approach, that leads to a three-stage structure per time-step cycle. These stages are composed of a Taylor-Galerkin scheme (Stage 1: two-step Lax-Wendroff, predictor-corrector) which solves simultaneously for velocity and stress; and a pressure-correction method (Stage 2 and 3), to enforce incompressibility and ensure second-order accuracy in time [21, 22, 23, 24]. The diffusion terms in the momentum equation are treated in an implicit manner, in order to enhance stability, whilst avoiding the computational overhead that would result from a fully-implicit scheme. From Stage 1 and 3, "augmented"-Galerkin Mass matrix-vector equations emerge, that are solved via an efficient element-byelement Jacobi scheme. This procedure requires only a handful of iterations

(five, typically). As for solution of the discrete pressure-difference equation of Stage 2, this is handled through a direct Choleski-decomposition procedure. All these aspects are expanded upon within $[16,23]$ and references therein.

The domain is discretised into six-node, triangular finite elements cells, Fig. 2a. Velocity components are interpolated via quadratic shape functions, using vertex and mid-side nodal values. Pressure is represented in a linear fashion, 
based on vertex nodal-values alone. These two field variables are computed with the $f e$-scheme outlined above.

In the hybrid $f e / f v$ context, we appeal to a cell-vertex finite volume $(f v)$ approach to spatially discretise the stress constitutive equations of Stage 1 . The relevant notions that this choice brings for conservation laws are consistency, upwinding (Fluctuation-Distribution, FD), linearity preservation and/or positivity. Such FD upwinding-schemes are known to be accurate for hyperbolic equations, and suffer less computational overhead compared to their finite element counterparts. Furthermore, the current hybrid implementation naturally integrates the $f v$-scheme within the $f e$-backbone, outlined above. This is achieved through the creation of four $f v$ triangular-subcells per parent $f e-$ triangle (Fig. 2a), reminiscent of the subcell-reference implementation of Marchal and Crochet [25] in the fe-context. Stress interpolation is equivalent to linear on the $f v$-subcell. Consequently, nodal stress values coincide across the two interpolation schemes, minimising projection error. Conversely, the velocity-field provided by the $f e$-section of the scheme is readily accessible to the $f v$-subsection, through vertices and mid-side nodal-values. This spatial embedding naturally meets compatibility constraints upon stress and velocity gradient fields, enhanced by gradient recovery strategies upon velocity fields.

\subsection{Finite volume nodal-update}

We proceed to recall briefly the finite volume methodology utilised here, details of which can be found in [14]. The stress nodal-update is based upon a cellvertex fluctuation-distribution (FD) procedure on each $f v$-subcell $T$, combined with a contribution from a Median-Dual-Cell (MDC) sub-area, see Fig. 2. The former, represented by FD-coefficient $\alpha_{l}^{T}$, allocates the flux $\left(R_{T}\right)$ and source $\left(Q_{T}\right)$ discrete residuals to the vertices $(l)$ of each $f v$-triangle $T$, according to an upwinding strategy (FD, see on). The MDC-contribution appends a uniform distribution of $R_{\mathrm{MDC}}^{l}$ and $Q_{\mathrm{MDC}}^{l}$, to node $l$ within $T$, from the MDC control volume. The MDC is a non-overlapping sub-area, defined around each node $l$, by connecting $f v$-cell vertices, midside-nodes and centroids, as demonstrated in Fig. 2b. These concepts are illustrated best by representing the stress constitutive equation in the form:

$$
\begin{aligned}
\frac{\partial \boldsymbol{\tau}}{\partial t} & =-\nabla \cdot \mathcal{R}+\boldsymbol{Q} \\
\mathcal{R} & =\boldsymbol{u} \boldsymbol{\tau} \\
\boldsymbol{Q} & =\frac{1}{W e}\left(2 \frac{\mu_{1}}{\mu} \boldsymbol{d}-f \boldsymbol{\tau}\right)+\boldsymbol{L} \cdot \boldsymbol{\tau}+\boldsymbol{\tau} \cdot \boldsymbol{L}^{T},
\end{aligned}
$$

where, $\mathcal{R}$ and $\boldsymbol{Q}$ represent the continuous flux and source terms, respectively. 
To extract the discrete flux, source and time-terms employed and construct the nodal-update equation for a particular scalar stress-component $\tau$, we integrate Eqs.(5)-(7) over appropriate control volumes:

$$
\int_{\Omega_{l}} \frac{\partial \tau}{\partial t} d \Omega=\int_{\Omega_{T}} \boldsymbol{u} \cdot \nabla \tau d \Omega+\int_{\Omega_{l}} Q d \Omega
$$

We use two such control volumes: each $f v$-subcell ${ }^{2} T_{l}$ surrounding node $l\left(\Omega_{T}\right)$, and its Median-Dual-Cell associated with node $l\left(\Omega_{l}\right)$, see Fig. $2 \mathrm{~b}$. For each $f v$ triangle $T$ and node $l-\mathrm{MDC}^{T}$, this procedure leads to the volume-respective flux $\left(R_{T}, R_{\mathrm{MDC}}^{l}\right)$ and source $\left(Q_{T}, Q_{\mathrm{MDC}^{T}}^{l}\right)$ terms. These contributions, in turn, are combined over all $T_{l}$ surrounding node $l$, to devise our CT2-scheme steady-state nodal-update equation for $\Delta \tau_{l}^{n+1}=\tau_{l}^{n+1}-\tau_{l}^{n}$, see $[18,26]$ :

$$
\frac{\Delta \tau_{l}^{n+1}}{\Delta t}=\underbrace{\frac{\sum_{\forall T} \delta_{T} \alpha_{l}^{T}\left(R_{T}+Q_{T}\right)}{\hat{\Omega}_{F D}}}_{\text {Fluctuation distribution }}+\underbrace{\frac{\sum_{\forall T} \delta_{\mathrm{MDC}}^{T}\left(R_{\mathrm{MDC}^{T}}^{l}+Q_{\mathrm{MDC}^{T}}^{l}\right)}{\hat{\Omega}_{l}}}_{\text {Median-Dual-Cell }},
$$

where $\hat{\Omega}_{F D}=\sum_{\forall T} \delta_{T} \alpha_{l}^{T} \Omega_{T}$ and $\hat{\Omega}_{l}$ represents the area of the whole MDC surrounding node $l$. The parameters $\delta_{T}$ and $\delta_{\mathrm{MDC}}^{T}$ are those used in [28]; that is, $\delta_{\mathrm{MDC}}^{T}=1$ and $\delta_{T}=\kappa$, with $\kappa=\xi / 3$ if $|\xi| \leq 3$ and 1 otherwise $^{3}$. Here, $\xi=W e(a / h)$, where $a$ represents the magnitude of an average advection-velocity within each $f v$-cell $T$, and $h$ the square-root of the area of that $f v$-cell. There are two important aspects in this nodal-update: first, the consistent distribution of flux and source terms, both under fluctuationdistribution and MDC $[19,28]$; second, the arealess nature of the equation that reflects consistency in area-weighting. This CT2-approach has proved highlystable and accurate at steady-state, as demonstrated in [18] for the benchmark 4:1 contraction flow and Oldroyd-B model-fluids. Note, consistency in areaweighting adopts and extends upon suggestions of Hubbard and Roe [29], re time-consistency for pure convection problems. Here, these are applied to situations with complex, and often dominant source-terms (see Aboubacar and Webster [26]).

$\overline{2}$ Note, it is our experience that non-conservative flux integration is paramount to ensure high-order accuracy at steady-state, see Aboubacar and Webster [26] and Chandio and Webster [27].

3 Different combinations of $\delta_{T}$ and $\delta_{\mathrm{MDC}}^{T}$ may be employed to yield alternative update strategies. For example, $\delta_{T}=1$ and $\delta_{\mathrm{MDC}}^{T}=0$ would lead to a pure FDapproach, not suitable for source-dominated problems. 


\subsection{Time consistency}

Given that the present work seeks to investigate transient flow properties, we have extended the CT2-scheme in order to embrace time-consistency in a rigorous manner. First, we consider separately the two right-hand-side contributions from Eq. 9. For each $f v$-triangle $T$ (area $\Omega_{T}$ ), and node $l$ with associated MDC within $T$ (area $\hat{\Omega}_{l}^{T}$ ), this leads to

$$
\frac{\Omega_{T}}{\Delta t} \alpha_{l}^{T}\left[\Delta \tau^{n+1}\right]_{l}=\alpha_{l}^{T}\left(R_{T}+Q_{T}\right)=\alpha_{l}^{T} b^{T}, \quad \text { on } \Omega_{T},
$$

if Fluctuation-Distribution is considered alone, and to

$$
\frac{\hat{\Omega}_{l}^{T}}{\Delta t}\left[\Delta \tau^{n+1}\right]_{l}=\left(R_{\mathrm{MDC}^{T}}+Q_{\mathrm{MDC}^{T}}\right)^{l}=b_{l}^{\mathrm{MDC}^{T}}, \quad \text { on } \mathrm{MDC}
$$

when only Median-Dual-Cell treatment is present. We propose to blend these two contributions, using the parameters $\delta_{T}$ and $\delta_{\mathrm{MDC}}^{T}$, generating, as a result, CT3-scheme. After summation over all $f v$-subcells surrounding node $l$, we obtain

$$
\left[\sum_{T_{l}} \Omega_{T} \delta_{T} \alpha_{l}^{T}+\sum_{\mathrm{MDC}_{l}} \delta_{\mathrm{MDC}}^{T} \hat{\Omega}_{l}^{T}\right] \frac{\Delta \tau_{l}^{n+1}}{\Delta t}=\sum_{\forall T_{l}} \delta_{T} \alpha_{l}^{T} b^{T}+\sum_{\forall \mathrm{MDC}_{1}} \delta_{\mathrm{MDC}^{T}} b_{l}^{\mathrm{MDC}^{T}}
$$

Note, a uniform distribution $\alpha$-scheme $\left(\alpha_{i}^{T}=1 / 3, \forall i, T\right)$ would generate an area factor of $\hat{\Omega}_{l}$ on time-terms, thus reverting to a pure MDC-scheme if $\delta_{\mathrm{MDC}}^{T}=1-\delta_{T}$.

Using the channel flow problem, we have experimented with alternative forms for $\delta_{T}$ (e.g. indexed to the maximum-/or average-velocity in the flow, smooth dependence upon cross-channel velocity profile). None of these improved upon the standard choice used in CT2, (see Eq. (9) above). Indeed, the dependence of $\delta_{T}$ on local average-velocity presents an interesting feature: its value vanishes in slow-flow regions (typically at the vicinity of no-slip shear-boundary), where source-terms would play a dominant role (steep velocity and stress-gradients); in contrast, $\delta_{T}$ tends to unity in fast-flow regions, in which resulting large velocity values would favour the prominence of flux-terms (better handled via FD). This provides a natural hint as to a complementary setting for the MDC weighting parameter, in the form $\delta_{\mathrm{MDC}}^{T}=1-\delta_{T}$. Such $\delta_{\mathrm{MDC}}^{T}$ selection has brought about significant improvement in transient accuracy for CT2-type schemes. The approach-CT3 is superior in updates to that of CT2-scheme (see [14] for details), and for the most part, CT3 is practically independent of $\delta_{\mathrm{MDC}}^{T}$ selection. This difference in parameter-setting optimality follows from 
the respective designs of both schemes: CT2 is essentially an additive-type approach based on arealess weighting of rhs-terms. In contrast, CT3 is based upon the blending of two contributions, under lhs-term area consideration.

\subsection{Fluctuation-Distribution schemes}

The fluctuation-distribution (FD) coefficients $\alpha_{l}^{T}$, in Eqs. ( 9) and (12), are extracted via compact-stencil upwinding techniques. These coefficients update the solution of a scalar advection equation by splitting cell-flux (and/or source) variations to the vertices of a $f v$-subtriangle. There is a variety of such FDschemes, see [29, 30, 31, 32], with properties such as positivity and linearity preservation. Here, we retain the Low Diffusion B (LDB) scheme. This exhibits optimal accuracy in tracking transient solutions, as demonstrated in [14]. The LDB- $\alpha_{i}^{T}$ coefficients are defined as

$$
\begin{array}{r}
\alpha_{i}^{T}=\frac{\sin \gamma_{1} \cos \gamma_{2}}{\sin \left(\gamma_{1}+\gamma_{2}\right)}, \\
\alpha_{j}^{T}=\frac{\sin \gamma_{2} \cos \gamma_{1}}{\sin \left(\gamma_{1}+\gamma_{2}\right)}, \\
\alpha_{k}^{T}=0,
\end{array}
$$

where $\gamma_{1}$ and $\gamma_{2}$ represent the angles substending the cell advection-velocity $\boldsymbol{a}$ (averaged) in a given $f v$-subcell $T$ with vertices $i, j, k$. Note, the closer the advection-velocity is to a given side, the greater the contribution to the downstream vertex of that side, without any dependence upon the magnitude of the local velocity.

\section{Discussion of results}

\subsection{Start-up channel flow}

We proceed to demonstrate the transient accuracy of the CT3 scheme discussed above (section 3.2), by applying it to the start-up planar Poiseuille flow (see section 2). To this end, we compare the evolution of stress-components $\left(\tau_{x x}\right.$ and $\left.\tau_{x y}\right)$ against the analytical solution, at sample points of identical $y$-levels, away from the boundaries. We consider two such stations, one located at the shear-boundary and the other in core-flow, as depicted in Fig. 1a. Starting with the major stress-component $\tau_{x x}$, Figs. 3a,b reveal the complex behaviour of the analytical solution (overshoots/undershoots), as it evolves 
towards a steady-state. This demonstrates the relevance of the model problem, in establishing transient accuracy for numerical algorithms in general. We contrast two settings of parameter $\delta_{\mathrm{MDC}}^{T}$, namely $\delta_{\mathrm{MDC}}^{T}=1$, and the dynamic alternative $\delta_{\mathrm{MDC}}^{T}=1-\delta_{T}\left(\right.$ since $\left.\delta_{T}=\delta_{T}(\boldsymbol{x}, t)\right)$. Under both parameter settings, CT3-scheme follows the analytical form closely at both stations, with an excellent reproduction of phase-matching. Hence, we have demonstrated the relative independence of CT3-scheme to $\delta_{\mathrm{MDC}}^{T}$-choice, and $\delta_{\mathrm{MDC}}^{T}=1$ is adopted henceforth. It is also worth noting that the MDC-contribution is an important stabilising factor of the scheme [14, 28], and particularly so for source-dominated flows.

The agreement between the numerical and the analytical solution is even better on $\tau_{x y}$ (Figs. 4a,b) and $\mathcal{U}_{x}(y)$ (not reported, for the sake of conciseness). In the case of $\tau_{x y}$, this is a reflection of the linear subcell-interpolation of stress that matches the analytical form. Matching to the analytical solution is achieved likewise in velocity (calculated via the $f e$-parent-element, under a quadratic representation).

\subsection{Application to a complex flow}

By way of extension to a more realistic setting, we apply the optimal scheme, derived in the previous section [LDB,CT3, $\left.\delta_{\mathrm{MDC}}^{T}=1\right]^{4}$ to a start-up 4:1 planar contraction creeping flow. Here, we focus upon the influence of boundary conditions settings (time-dependent versus static) on flow-structure.

Commencing with the time-dependent boundary conditions scenario, Fig. 5 charts the centreline-velocity against time, monitored at the inlet for $W e=$ $0.3, W e=1$ and $W e=2$. For the sake of clarity, we restrict the plotting timeinterval to $0 \leq t \leq 5$ units. Steady-state is achieved at $t=3.99,14.0,59.7$ units for $W e=0.3,1,2$, respectively. We note, the similarity of transients at different $W e$-values, with more pronounced overshoots/undershoots and exaggerated onset of peaks with increasing $W e$. Peaks are associated with each overshoot/undershoot in the solution, so that we identify five such peaks for $W e=0.3$; the first $(\mathrm{P} 1)$, corresponds to the first overshoot, and the last $(\mathrm{P} 5)$ to steady-state. Note, the fourth peak is faint, and located at $t=2.11$ units. In addition, we report in Figs. 6,7, the inlet transient stress-profiles for $\tau_{x x}$ and $\tau_{x y}$, respectively. These are monitored at the wall, at the same $W e$-levels as those employed for velocity. The succession of gradually-dampened overshoots/undershoots is present in both stress components. However, some differences are apparent. For example, $\tau_{x x}$-levels increase with increasing $W e$ (both first peak-magnitude and steady-state plateau-values). In contrast for

$\overline{4}$ Note on stability of schemes (transient from rest, for each $W e$-value): $W e_{\text {crit }}=1.7$ for $\left[\mathrm{LDB}, \mathrm{CT} 3, \delta_{\mathrm{MDC}}^{T}=1-\delta_{T}\right]$ and $W e_{\text {crit }}=2.0$ for $\left[\mathrm{LDB}, \mathrm{CT} 3, \delta_{\mathrm{MDC}}^{T}=1\right]$. 
$\tau_{x y}$, the first peak-magnitude is affected barely by $W e$-level, but takes longer time to reach as $W e$ increases, and the steady-state asymptote is identical for all three $W e$ : this is a reflection of the constant-viscosity nature of the Oldroyd-B model (note, variable-values are non-dimensional).

We begin with a relatively low $W e$-level, and investigate flow-structure evolution. Fig. 8 demonstrates the transient evolution of streamlines for $W e=0.3$, from a short-time ( $t=0.33$ units) after start-up of flow, to ultimate steadystate $(t=3.99$ units). Provocative evolutionary flow features may be identified. A salient-corner vortex builds-up gradually to P1 (first-overshoot, $\mathrm{t}=0.33$ units). As centreline-velocity gradually declines (cf. Fig. 5), a large secondaryvortex appears (half-way between P1 and P2 time). This extends way back upstream, signalling the appearance of a reverse-flow region (near the upstreamwall). As centreline-velocity rises towards P3 time, reverse-flow is neutralised progressively, till it disappears altogether. This occurs half-way between P2 and $\mathrm{P} 3$, at around $t=1.23$ units, where salient-corner vortex has almost disappeared. From this point-onwards, salient-corner vortices start to grow oncemore (up to circa $t=1.82$ units), before gradually decreasing towards its steady-state form. The correlation is manifest between such time-evolution, and the oscillatory-development of the inlet (analytical) boundary states in velocity and stress.

Next, we proceed to contrast this scenario against that of time-independent boundary conditions, again with flow initiated from rest and at the same level of $W e$. In this case, a velocity-profile against time at a centreline sample-point, somewhat removed from the inlet and the contraction-plane, would manifest a shocked-response; hence, reflecting response to the impulsive shock. In contrast and for the time-dependent scenario, a sample-point velocity profile-evolution is identical to that at the inlet (undisturbed flow away from the contraction). Streamline-patterns are reported in Fig. 9 for the static inlet flow-setting. In contrast to transient inlet-setting, we observe a monotonic rise in salientcorner vortex-size, up to time 1.82 units. This is followed by a monotonic, albeit small, decrease to steady-state. No complex transient flow-structure emerges. This evolutionary flow-pattern (first increase/then decrease) is similar to that reported by Mompean and Deville [9] for the same problem, under identical steady boundary conditions ${ }^{5}$. We find the same salient-corner vortex length ( $X=0.19$ units) at steady-state. Also, we note that steadystate streamline maximum-intensities in the recirculation region concur within $0.9 \%$, between the present transient and static (steady) inlet-setting results. In the main, steady-states all superimpose. Nevertheless, the principal point is made clearly: true-transients are observed only in the time-dependent inletflow problem. This is in direct juxtaposition with previous claims.

$\overline{5}$ These authors used a Deborah number of 0.3 , defined as $\lambda * U / L$, and a Reynolds number of 0.01 (creeping flow). 
So far, the discussion has focused on a $W e$-level that is essentially a perturbation beyond that of the Newtonian state. Next, we proceed to interrogate flow-structure evolution at higher levels of elasticity, for both flow settings. We choose $W e=2$, again initiating flow from a quiescent state. First, we commence with time-evolving inlet-flow boundary conditions. Figs. 10-11 chart streamlines against time, from early development ( $t=0.2$ units) until steadystate $(t=59.7$ units). The unique evolutionary flow features observed for the less-elastic fluid $(W e=0.3)$ are replicated, and amplified. Here again, transitions are dictated by the inlet-velocity peaks. A salient-corner vortex becomes conspicuous at P1 (first-overshoot). The reverse-flow region that appears half-way between P1 and P2, now expands internally towards core-flow. This secondary-vortex reaches a maximum width at P2 (appearance of firstundershoot), covering about half the contraction-wall, and is accompanied by a lip-vortex and a salient-corner vortex. Subsequently, as the centreline-velocity increases towards $\mathrm{P} 3$, there is a gradual neutralisation of back-flow, until its total disappearance between P2 and P3 ( $t=2.90$ units). At this juncture, we observe an eye-like, highly-localised, secondary-vortex. Note, both salient and lipvortices have subsided by this station in time. From this point-onwards (Fig. 11), both lip and salient-corner vortices start to grow oncemore, and evolve towards their steady-state forms, with only minor fluctuations in their respective sizes. These dynamic fluctuations in flow-structure (increase/decrease) reflect the gradually dampened oscillations in the inlet velocity-profile, that die away as the velocity asymptotes towards its steady-state plateau-value.

By way of contrast, we next focus on the fixed (steady) inlet boundary conditions flow-scenario (Figs. 12-13), at the elevated $W e$-level, $W e=2$. As in the $W e=0.3$-case, salient-corner vortex-size increases monotonically (to around 3 time units), declining thereafter towards its steady-state representation. The main differences in evolutionary patterns between the two levels of $W e$ lie, for $W e=2$, in the larger salient-corner vortices generated in the march towards steady-state, and hint of the appearance of a lip-vortex. The strength and evolution of this lip-vortex, however, exhibits a distinct difference in the more realistic, dynamic flow setting. It commences earlier in the time-dependent boundary condition case (around 2 units), and because of transient dynamics about the re-entrant corner (see Figs. 10- 11). In the time-independent boundary condition case, lip-vortex dynamics is much less conspicuous. Experimental evidence on lip-vortices and their dynamics is covered in McKinley et al. [33] and Boger [34] for circular geometries, and in Evans and Walters $[35,36]$ for planar instances.

The dynamic evolution and emerging stress field structure is reported via transient stress-contours in Fig. 14 and Fig. 15, for first normal stress-difference $\left(N_{1}=\tau_{x x}-\tau_{y y}\right)$ and shear-stress, respectively ( $W e=2$, elastic components alone). The times reported are identical to those referred to for streamlines in Figs. 10-11. It is clear that the steady-state structure, around and after the 
re-entrant corner is established somewhat earlier in normal-stress than shearstress. To give some appreciation of this, we report that the stress boundarylayer reaches its steady-state structure (length and width) in $N_{1}$ by around 20 time units, whilst this is delayed to around 60 time units in $\tau_{x y}$ (see Fig 16). Moreover, upstream of the contraction, the change in flow-structure (from unidirectional shear-flow to reversed-flow) is reflected in the shear-stress, Fig. $15 \mathrm{c}-\mathrm{f}$. In contrast, the first normal stress-difference re-echoes the curvature of the emerging salient-corner vortex. This is due to dependence upon the $\tau_{y y}$-field, as demonstrated by Aboubacar et al. [12], in a steady-state context. Experience with time-accuracy, through time-step reduction (by one order of magnitude), reveals that all solutions reported above are sufficiently converged, so that no differences are detected to such precision.

\section{Conclusions}

We have demonstrated that the nature of the boundary conditions has a significant impact on the transient flow-structure in a start-up 4:1 contraction flow for Oldroyd-B model fluids. In contrasting the evolutionary streamlines of static inlet boundary conditions against those for time-dependent dynamic instances, we have found radical differences in flow structure, as the system evolves from rest towards an identical, ultimate steady-state. In a fixedboundary conditions scenario, we have reproduced the simple increase/decrease of salient-corner vortex-size reported in the literature. However, complex features have emerged in flow-structure in the transient boundary conditions scenario, notably the appearance of a large secondary-flow at early evolutiontimes. Moreover, at significant levels of Weissenberg number, this complex flow-structure exhibit a dynamic development of a lip-vortex, generated by the extension of the early-time secondary-flow, up to and beyond the reentrant corner. We have established that this elaborate evolutionary flowstructure correlates with the imposition of transient inlet-boundary conditions. Therefore, some doubt is cast upon the common assumption that static inlet-boundary conditions do not influence transient flow-structure near the contraction zone, irrespective of distant entry and exit die-stations from the contraction plane. Also, we have shown that it is the shear-stress that governs the long-time establishment of the steady-state regime, through post-corner downstream-wall stress boundary-layers. In addition, transient shear-stress fields reflects the dynamic changes in flow-structure far back along the upstream wall. These two features essentially bear out the shearing nature of the flow in these regions. In contrast and in the salient-corner vicinity, it is the elongational stress $\left(\tau_{y y}\right)$ that characterises the dynamic-flow vortex-patterns. This investigation has been possible only through the development of an ac- 
curate transient, hybrid finite volume/element algorithm, which we commend accordingly.

\section{Acknowledgments}

The authors gratefully acknowledge the partial financial support of TMR/EU Network grant for MA and EPSRC for HRTJ during the course of this research. Also, we are indebted to our Institute of Non-Newtonian Fluid Mechanics colleagues, Professors Walters and Phillips for their many helpful contributions. 


\section{References}

[1] R. Keunings. Simulation of viscoelastic flow. In C.L. Tucker, editor, Computer Modeling for Polymer Processing, pages 404-469, Hanser, Munich, 1989.

[2] F.T.P. Baaijens. Mixed finite element methods for viscoelastic flow analysis: a review. J. Non-Newtonian Fluid Mech., 79:361-385, 1998.

[3] K. Walters and M.F. Webster. The Distinctive CFD Challenges of Computational Rheology. ECCOMAS Swansea 2001, to appear in Int. J. Num. Meth. Fluids, 2001.

[4] N. El KISS and J-M. PIAU. Enhancing processability of polymer melts during extrusion. In Proc. 6th European Conf. Rheol., pages 3-8, Erlangen, Germany, 2002. eds. H. Münstedt, J. Kaschta and A. Merten.

[5] J.-M. Piau, S. Nigen, and N. El Kissi. Effect of die entrance filtering on mitigation of upstream instability during extrusion of polymer melts. $J$. Non-Newtonian Fluid Mech., 91:37-57, 2000.

[6] F. Olsson. A solver for time dependent viscoelastic fluid flows. J. NonNewtonian Fluid Mech., 51:309-340, 1994.

[7] P. Wapperom, R. Keunings, and V. Legat. The backward-tracking lagrangian particle method for transient viscoelastic flows. J. NonNewtonian Fluid Mech., 91:273-295, 2000.

[8] G.B. Bishko, O.G. Harlen, T.C.B. McLeish, and T.M. Nicholson. Numerical simulation of the transient flow of branched polymer melts through a planar contraction using the 'pom-pom' model. J. Non-Newtonian Fluid Mech., 82:255-273, 1999.

[9] G. Mompean and M.O. Deville. Recent developments in threedimensional unsteady flows of non-newtonian fluids. Revue de l'Institut Français du Pétrole, 51:261-267, 1996.

[10] M. Aboubacar, T.N. Phillips, H.R. Tamaddon-Jahromi, M.F. Webster, and A.J. Williams. Numerical Simulation of Contraction Flows for Boger Fluids using Finite Volume Methods. In Proc. 6th European Conf. Rheol., pages 279-280, Erlangen, Germany, 2002. eds. H. Münstedt, J. Kaschta and A. Merten.

[11] M. Aboubacar, H. Matallah, and M.F. Webster. Highly Elastic Solutions for Oldroyd-B and Phan-Thien/Tanner Fluids with a Finite Volume/Element Method: planar contraction flows. In press. J. NonNewtonian Fluid Mech., 2001, available as CSR17-2000.

[12] M. Aboubacar, H. Matallah, H.R. Tamaddon-Jahromi, and M.F. Webster. Numerical prediction of extensional flows in contraction geometries: hybrid finite volume/element method. J. Non-Newtonian Fluid Mech., 104:125-164, 2002.

[13] E.O.A. Carew, P. Townsend, and M.F. Webster. A Taylor-PetrovGalerkin algorithm for viscoelastic flow. J. Non-Newtonian Fluid Mech., 50:253-287, 1993.

[14] H.R. Tamaddon-Jahromi, M. Aboubacar, and M.F. Webster. Time- 
Dependent Algorithms for Viscoelastic Flow - finite element/volume schemes. submitted to Int. J. Num. PDE, 2002.

[15] N.D. Waters and M.J. King. Unsteady flow of an elastico-viscous liquid. Rheo. Acta, Band 9:Heft 3, 1970.

[16] E.O.A. Carew, P. Townsend, and M.F. Webster. Taylor-Galerkin algorithms for viscoelastic flow: application to a model problem. Num. Meth. PDE, 10:171-190, 1994.

[17] S. Xu, A.R. Davies, and T.N. Phillips. Pseudospectral Method for Transient Viscoelastic Flow in an Axisymmetric Channel. J. Num. Meth. PDE, 9:691-710, 1993.

[18] M. Aboubacar and M.F. Webster. A cell-vertex finite volume/element method on triangles for abrupt contraction viscoelastic flows. J. NonNewtonian Fluid Mech., 98:83-106, 2001.

[19] P. Wapperom and M.F. Webster. A second-order hybrid finiteelement/volume method for viscoelastic flows. J. Non-Newtonian Fluid Mech., 79:405-431, 1998.

[20] H. Matallah, P. Townsend, and M.F. Webster. Recovery and stresssplitting schemes for viscoelastic flows. J. Non-Newtonian Fluid Mech., 75:139-166, 1998.

[21] J. Donea. A Taylor Galerkin method for convective transport problems. Int. J. Num. Meth. Eng., 20:101-119, 1984.

[22] O.C. Zienkiewicz, K. Morgan, J. Peraire, M. Vandati, and R. Löhner. Finite elements for compressible gas flow and similar systems. In 7th Int. Conf. Comput. Meth. Appl. Sci. Eng., Versailles, France, 1985.

[23] D.M. Hawken, H.R. Tamaddon-Jahromi, P. Townsend, and M.F. Webster. A Taylor-Galerkin based algorithm for viscous incompressible flow. Int. J. Num. Meth. Fluids, 10:327-351, 1990.

[24] J. Van Kan. A second-order accurate pressure-correction scheme for viscous incompressible flow. SIAM J. Sci. Stat. Comput., 7, 3:870-891, 1986.

[25] J.M. Marchal and M.J. Crochet. A new mixed finite element for calculating viscoelastic flow. J. Non-Newtonian Fluid Mech., 26:77-114, 1987.

[26] M. Aboubacar and M.F. Webster. Development of an optimal hybrid finite volume/element method for viscoelastic flows. Under review, Int. J. Num. Meth. Fluids, available as CSR15-2000, University of Wales, Swansea,2000.

[27] M.S. Chandio and M.F. Webster. On consistency of cell-vertex finitevolume formulations for viscoelastic flow. In XIII Int. Cong. on Rheol., vol. 2, 208-210, Cambridge, 2000. eds. D.M. Binding, N.E. Hudson, J. Mewis, J-M Piau, C.J.S. Petrie, P. Townsend, M.H. Wagner and K. Walters; British Society of Rheology, Glasgow.

[28] P. Wapperom and M.F. Webster. Simulation for viscoelastic flow by a finite volume/element method. Comput. Meth. Appl. Mech. Eng., 180:281304, 1999.

[29] M.E. Hubbard and P.L. Roe. Multidimensional upwind fluctuation distribution schemes for scalar time dependent problems. Numerical Analysis 
report 1/98, Department of Mathematics, University of Reading, 1998.

[30] A. Jameson and D. Mavriplis. Finite volume solution of the twodimensional Euler equations on a regular triangular mesh. A.I.A.A, 24:611-618, 1986.

[31] K.W. Morton, P.I. Crumpton, and J.A. MacKenzie. Cell vertex methods for inviscid and viscous flows. Comput. and Fluids, 22:91-102, 1993.

[32] H. Deconinck, P.L. Roe, and R. Struijs. A multi-dimensional generalization of Roe's flux difference splitter for the Euler Equations. Comput. and Fluids, 22:215-222, 1993.

[33] G.H. McKinley, W.P. Raiford, R.A. Brown, and R.C. Armstrong. Nonlinear dynamics of viscoelastic flow in axisymmetric abrupt contractions. J. Fluid Mech., 223:411-456, 1991.

[34] D.V. Boger. Viscoelastic flows through contractions. Ann. Rev. Fluid Mech., 19:157-182, 1987.

[35] R.E. Evans and K. Walters. Flow characteristics associated with abrupt changes in geometry in the case of highly elastic liquids. J. NonNewtonian Fluid Mech., 20:11-29, 1986.

[36] R.E. Evans and K. Walters. Further remarks on the lip-vortex mechanism of vortex enhancement in planar-contraction flows. J. Non-Newtonian Fluid Mech., 32:95-105, 1989. 


\section{Figure captions}

Fig. 1. Finite element meshes.

a) Finite element 10x10 mesh, start-up Poiseuille flow, unstructured mesh.

b) Planar 4:1 section of contraction-flow mesh; Magnification around re-entrant corner.

Fig. 2. Spatial discretisation.

a) $f e$-cell with four $f v$ sub-cells. b) $f v$-control volume for node $l$, with MedianDual-Cell (MDC-shaded).

Fig. 3. $\tau_{x x}$ : [CT3-LDB] with various $\delta_{\mathrm{MDC}}$ settings, start-up Poiseuille flow. a) Boundary node. b) Interior node.

Fig. 4. $\tau_{x y}$ : [CT3-LDB] with various $\delta_{\mathrm{MDC}}$ settings, start-up Poiseuille flow. a) Boundary node. b) Interior node.

Fig. 5. 4:1 contraction, transient development of inlet centreline-velocity, [CT3$\left.\mathrm{LDB}, \delta_{\mathrm{MDC}}=1\right]$, Oldroyd-B.

Fig. 6. 4:1 contraction, inlet- $\tau_{x x}$ transient development at wall, [CT3-LDB, $\delta_{\mathrm{MDC}}=$ 1], Oldroyd-B.

Fig. 7. 4:1 contraction, inlet- $\tau_{x y}$ transient development at wall, [CT3-LDB, $\delta_{\mathrm{MDC}}=$ 1], Oldroyd-B.

Fig. 8. Time-dependent inlet boundary conditions: transient streamlines, 4:1 contraction, [CT3-LDB, $\delta_{\mathrm{MDC}}=1$ ], Oldroyd-B, $W e=0.3$.

Fig. 9. Static inlet boundary conditions: transient streamlines, 4:1 contraction, $\left[\mathrm{CT} 3-\mathrm{LDB}, \delta_{\mathrm{MDC}}=1\right]$, Oldroyd-B, $W e=0.3$.

Fig. 10. Time-dependent inlet boundary conditions: transient streamlines, 4:1 contraction, [CT3-LDB, $\delta_{\mathrm{MDC}}=1$ ], Oldroyd-B, $W e=2$ (early evolution). 
Fig. 11. Time-dependent inlet boundary conditions: transient streamlines, 4:1 contraction, [CT3-LDB, $\delta_{\mathrm{MDC}}=1$ ], Oldroyd-B, $W e=2$.

Fig. 12. Static inlet boundary conditions: transient streamlines, 4:1 contraction, [CT3-LDB, $\left.\delta_{\mathrm{MDC}}=1\right]$, Oldroyd-B, $W e=2$ (early evolution).

Fig. 13. Static inlet boundary conditions: transient streamlines, 4:1 contraction, $\left[\mathrm{CT} 3-\mathrm{LDB}, \delta_{\mathrm{MDC}}=1\right]$, Oldroyd-B, $W e=2$.

Fig. 14. Time-dependent inlet boundary conditions: transient $\tau_{x x}-\tau_{y y}$ fields, 4:1 contraction, [CT3-LDB, $\delta_{\mathrm{MDC}}=1$ ], Oldroyd-B, $W e=2$.

Fig. 15. Time-dependent inlet boundary conditions: transient $\tau_{x y}$ fields, 4:1 contraction, [CT3-LDB, $\delta_{\mathrm{MDC}}=1$ ], Oldroyd-B, $W e=2$.

Fig. 16. Downstream-wall, transient stress boundary layers development: $\tau_{x x}-$ $\tau_{y y}$ and $\tau_{x y}$ fields; 4:1 contraction, [CT3-LDB, $\delta_{\mathrm{MDC}}=1$ ], Oldroyd-B, $W e=2$. 
a) Finite element 10x10 mesh, start-up Poiseuille flow, unstructured mesh.

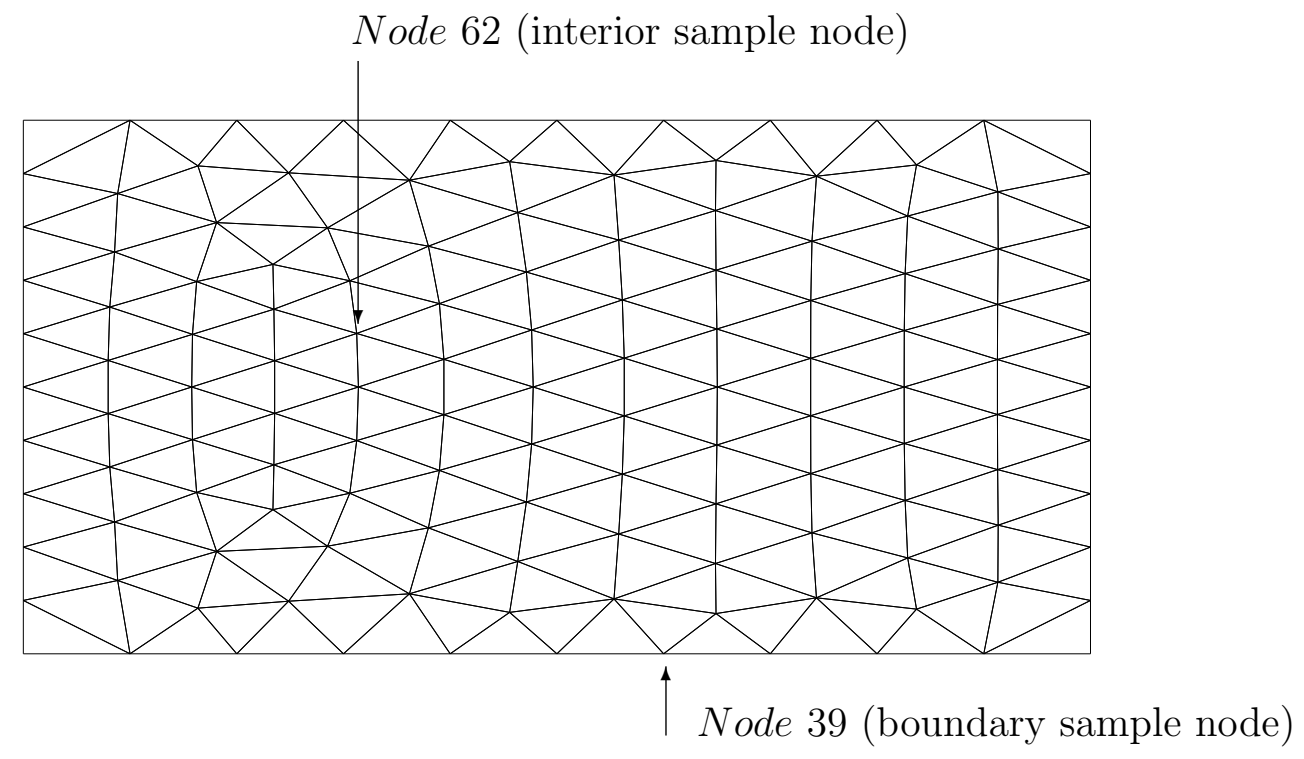

b) Planar 4:1 section of contraction-flow mesh; Magnification around re-entrant corner.

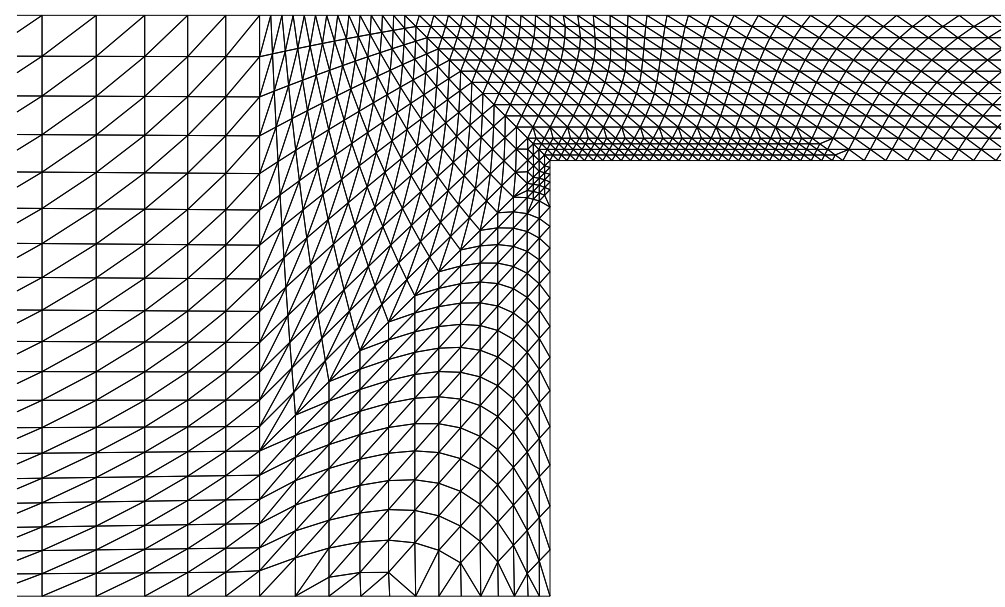

Fig. 1. Finite element meshes 


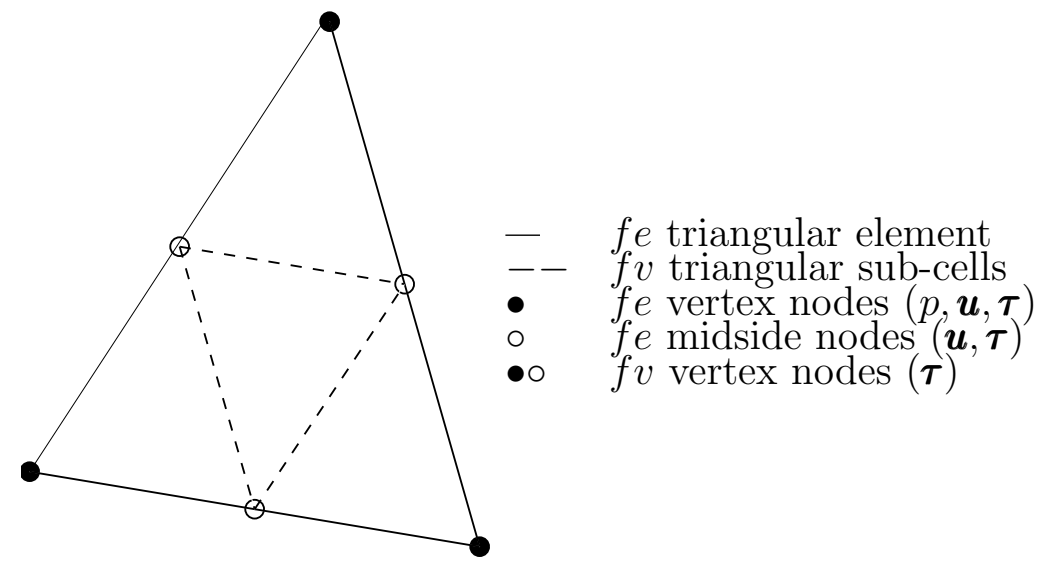

a) $f e$-cell with four $f v$ sub-cells.

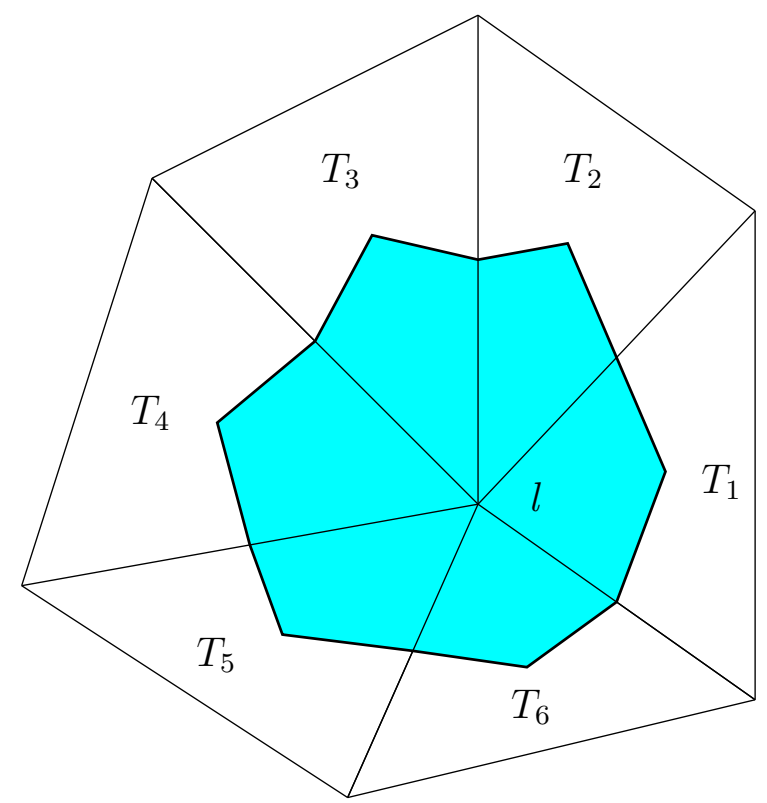

b) $f v$-control volume for node $l$, with Median-Dual-Cell (MDC-shaded).

Fig. 2. Spatial discretisation. 


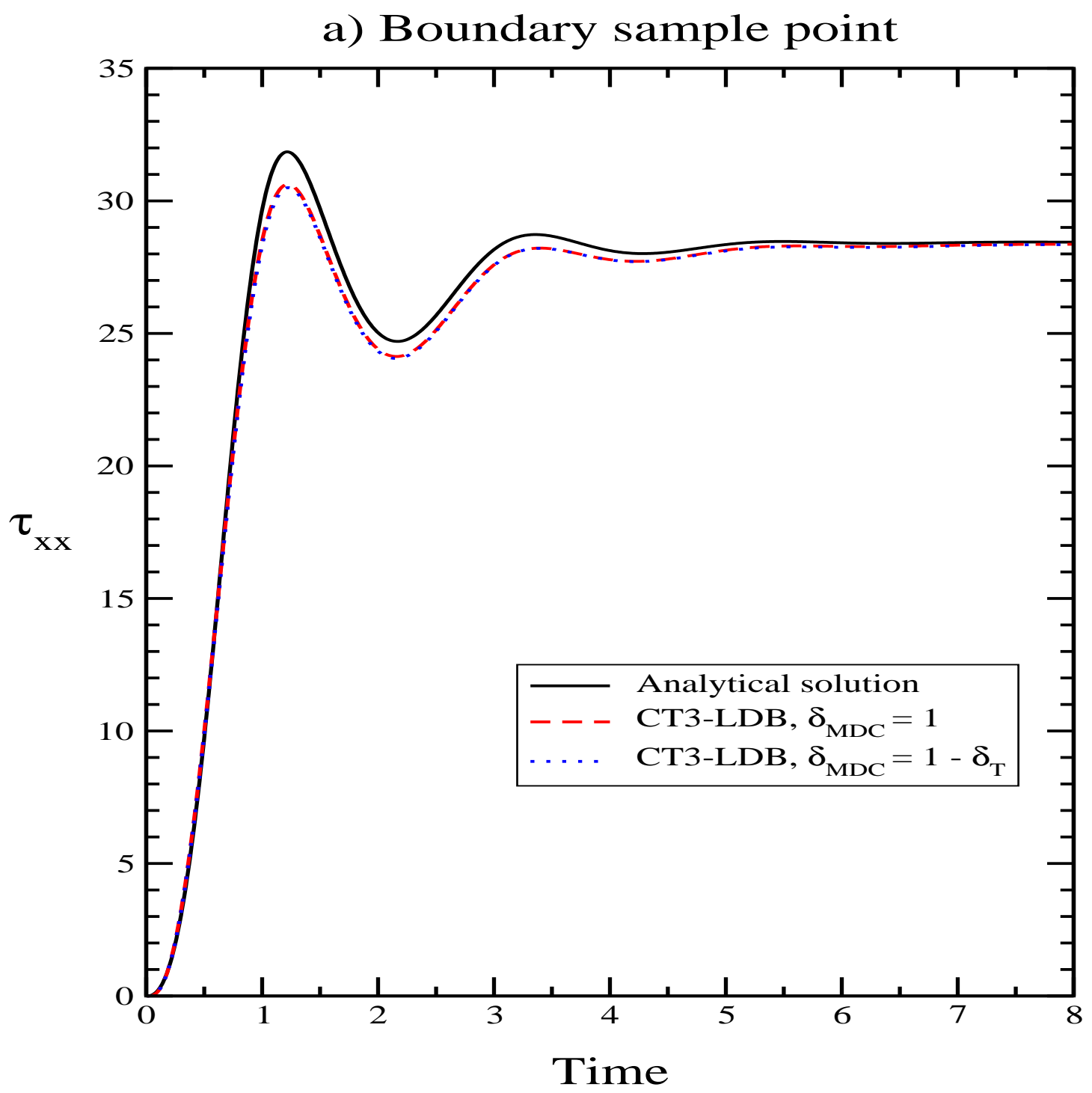

Fig. 3. a) Boundary node, $\tau_{x x}$ : [CT3-LDB] with various $\delta_{\mathrm{MDC}}$ settings, start-up Poiseuille flow. 


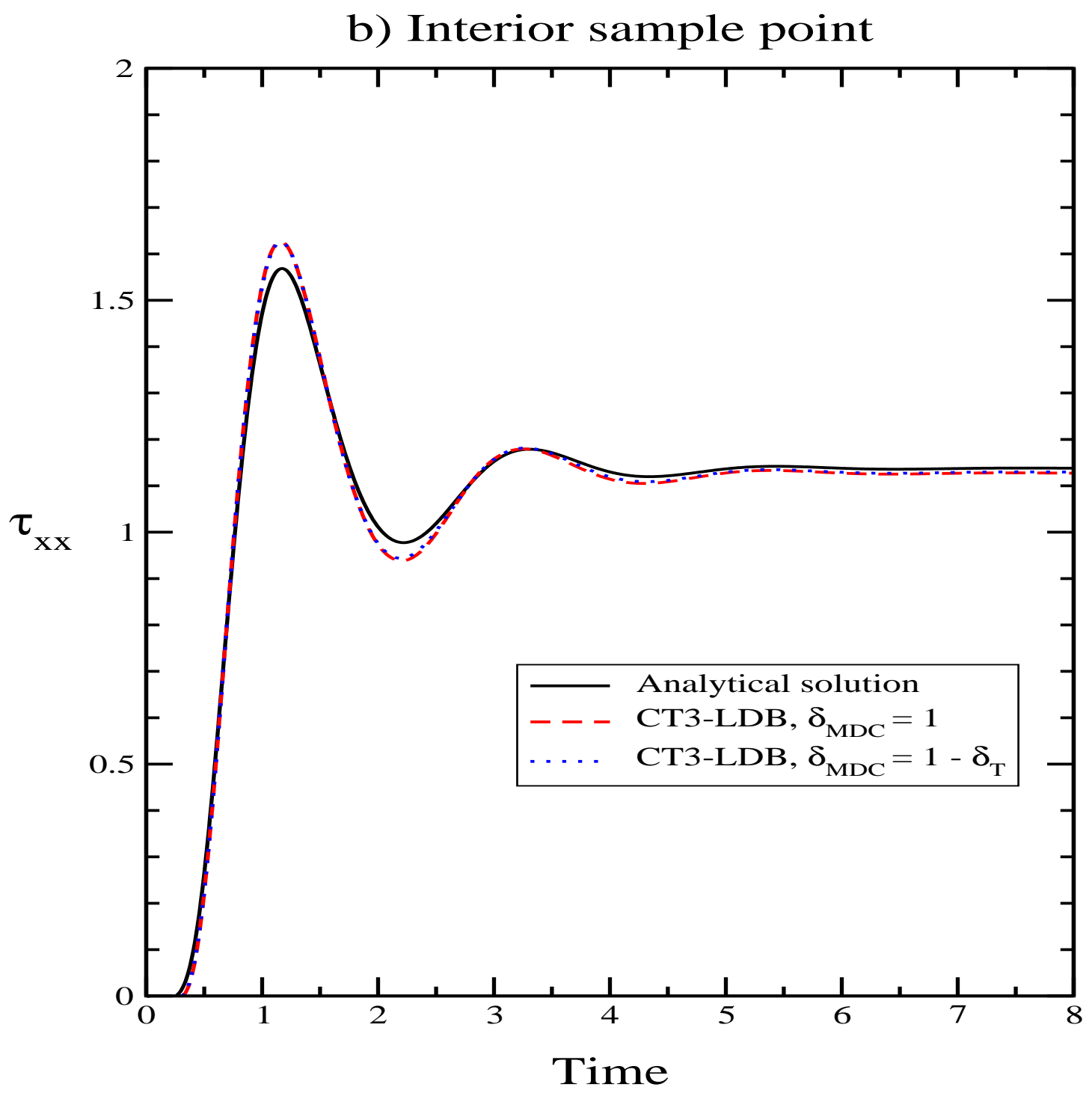

Fig. 3. b) Interior node, $\tau_{x x}$ : [CT3-LDB] with various $\delta_{\mathrm{MDC}}$ settings, start-up Poiseuille flow. 


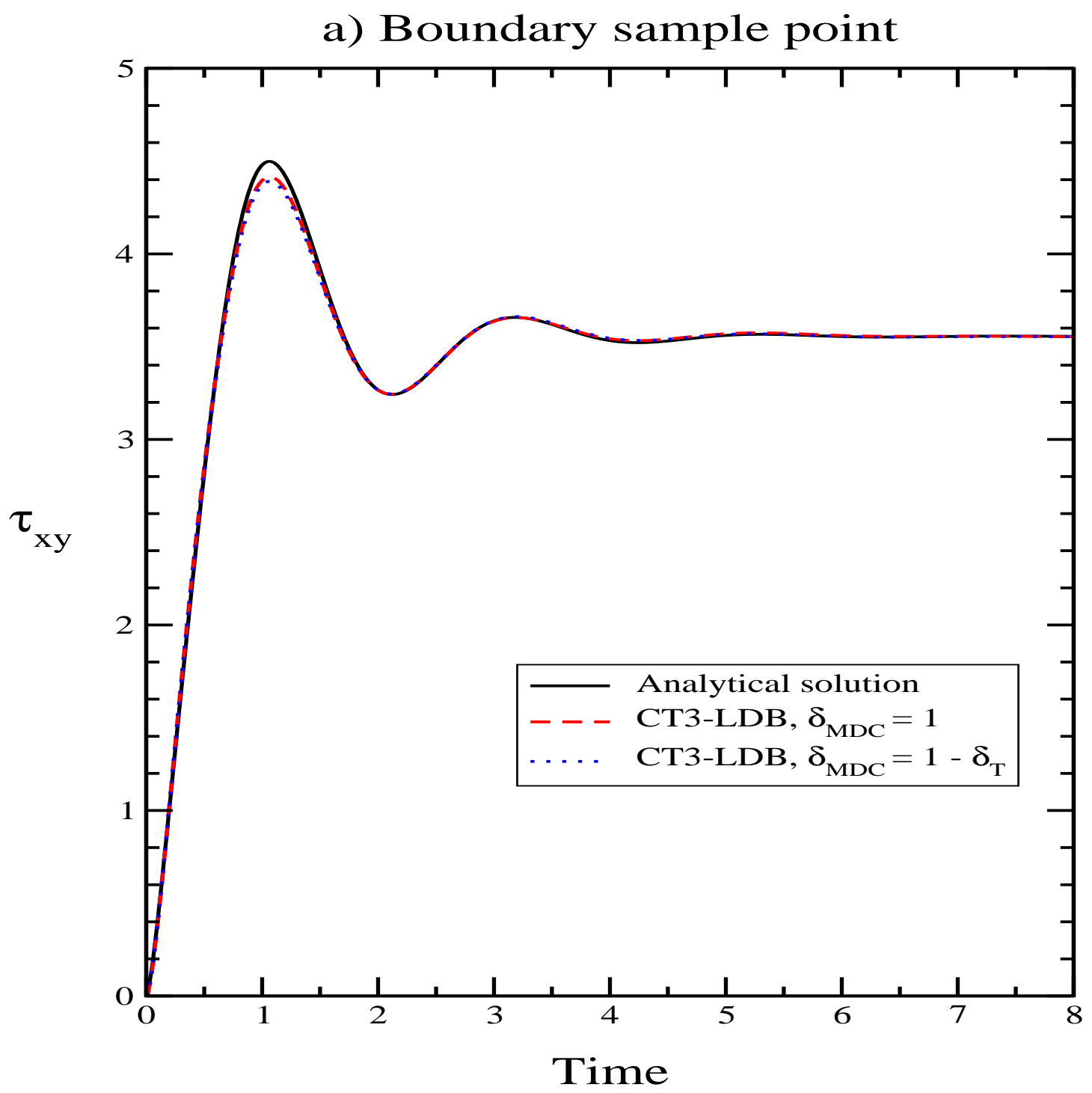

Fig. 4. a) Boundary node, $\tau_{x y}$ : $[\mathrm{CT} 3-\mathrm{LDB}]$ with various $\delta_{\mathrm{MDC}}$ settings, start-up Poiseuille flow. 


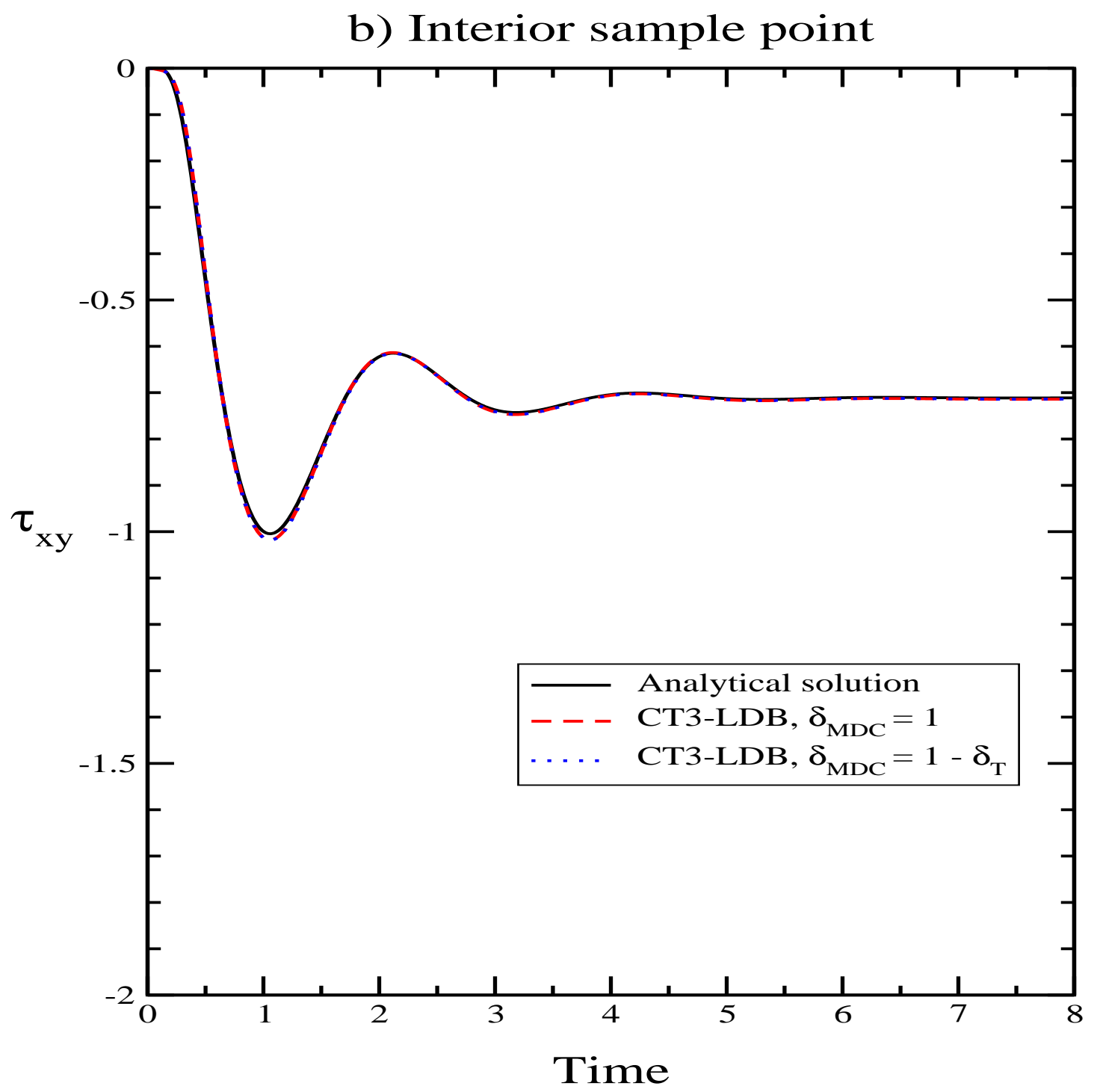

Fig. 4. b) Interior node, $\tau_{x y}$ : [CT3-LDB] with various $\delta_{\mathrm{MDC}}$ settings, start-up Poiseuille flow. 


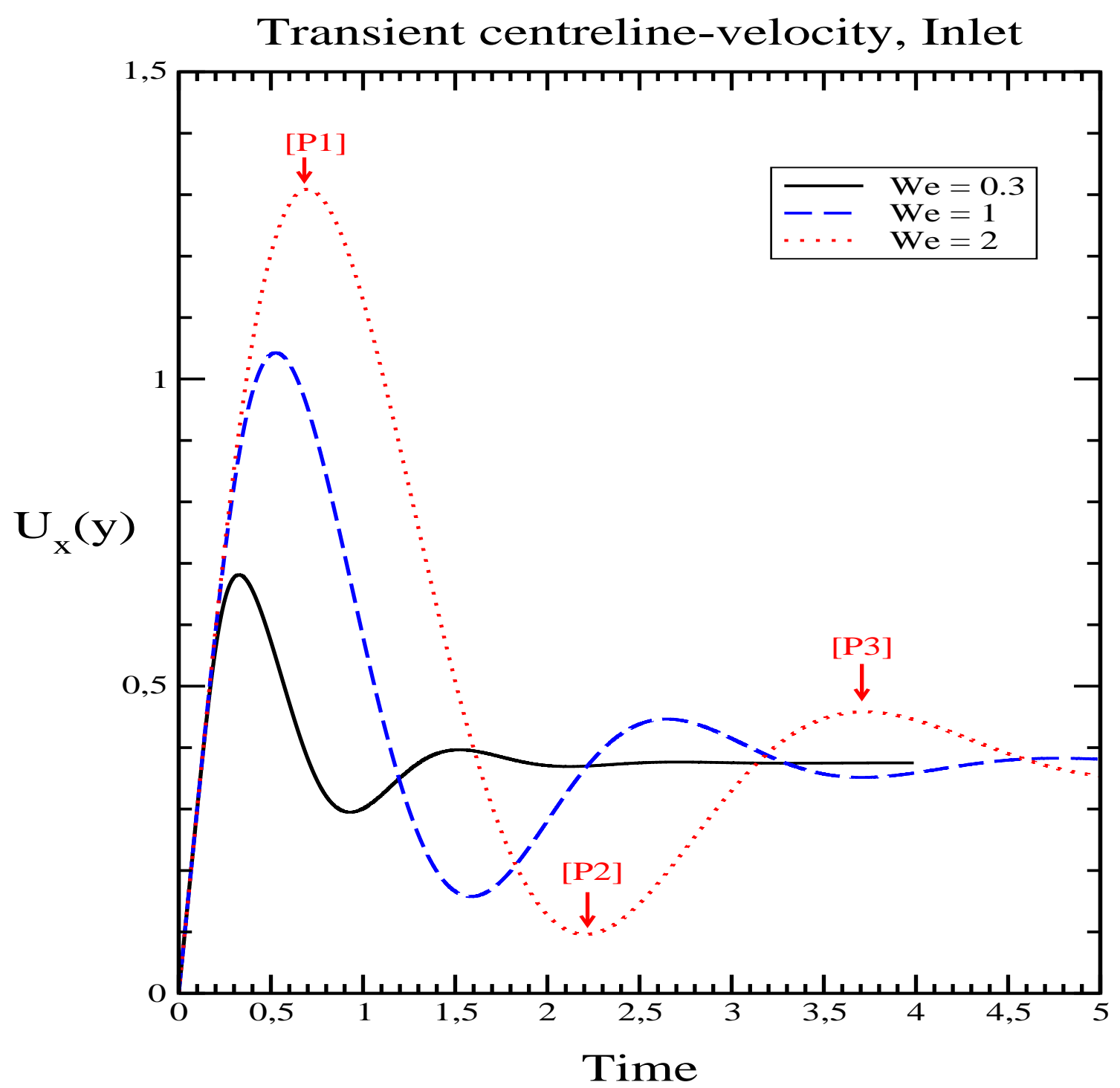

Fig. 5. 4:1 contraction, transient development of inlet centreline-velocity, $\left[\mathrm{CT} 3-\mathrm{LDB}, \delta_{\mathrm{MDC}}=1\right]$, Oldroyd-B. 


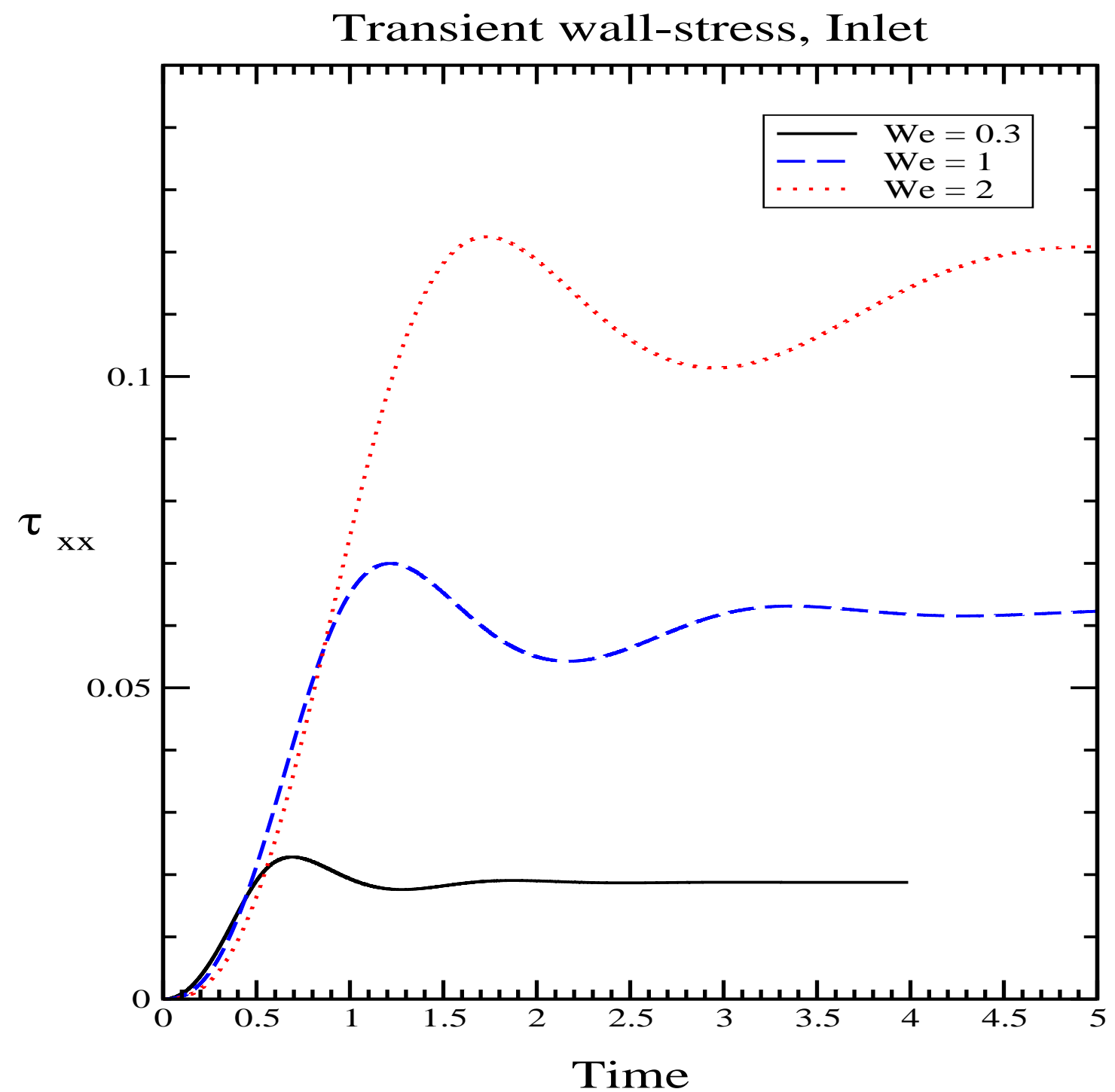

Fig. 6. 4:1 contraction, inlet- $\tau_{x x}$ transient development at wall, $\left[\mathrm{CT} 3-\mathrm{LDB}, \delta_{\mathrm{MDC}}=1\right]$, Oldroyd-B. 


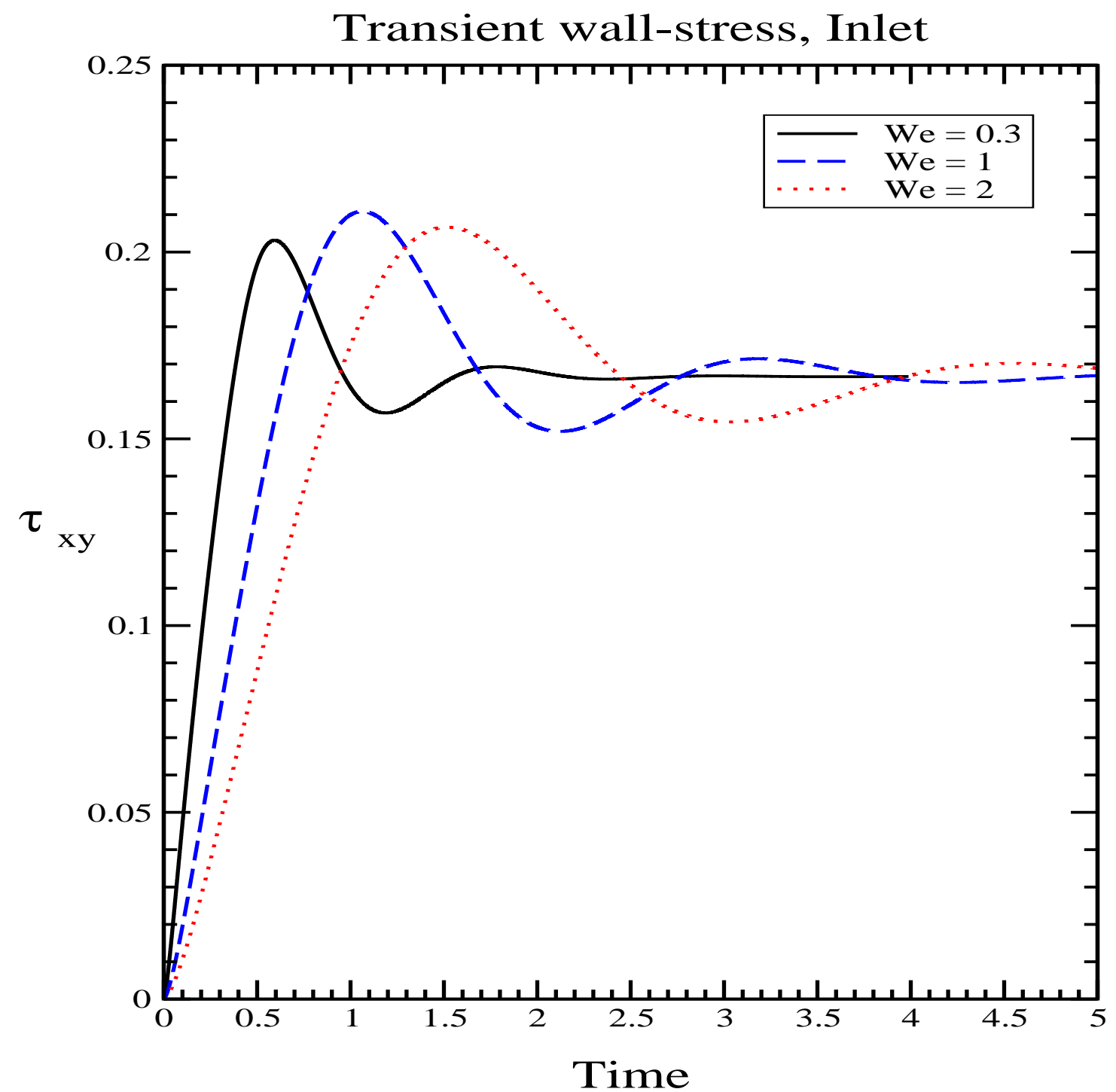

Fig. 7. 4:1 contraction, inlet- $\tau_{x y}$ transient development at wall, $\left[\mathrm{CT} 3-\mathrm{LDB}, \delta_{\mathrm{MDC}}=1\right]$, Oldroyd-B. 
a) $t=0.33$

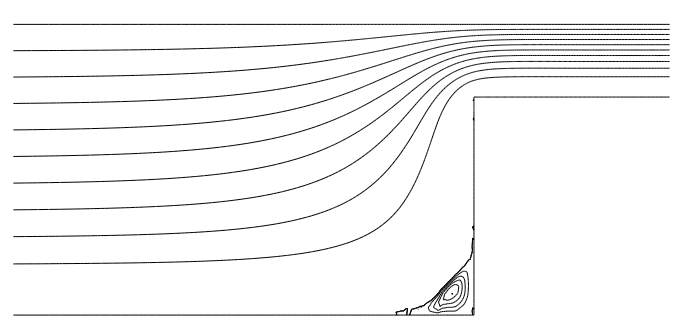

a) $t=0.63$

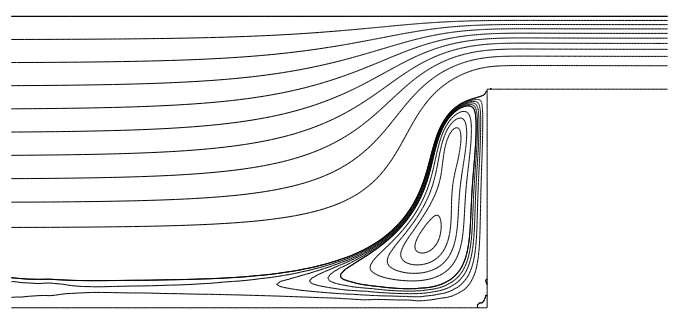

b) $t=1.23$

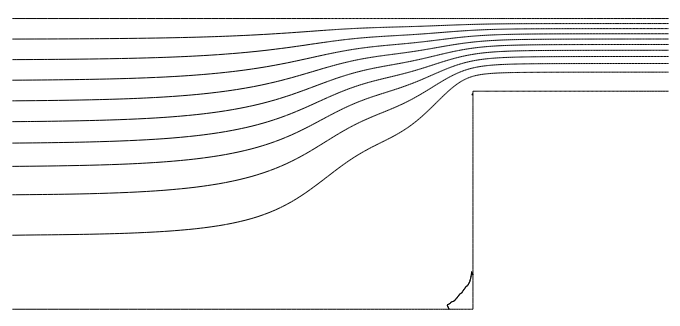

c) $t=1.82$

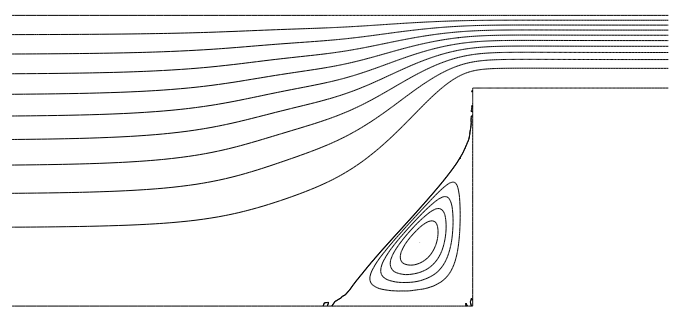

d) $t=3.10$

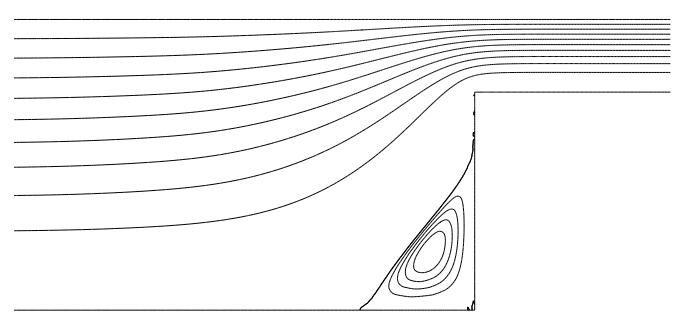

e) $t=3.99$ (Steady-state)

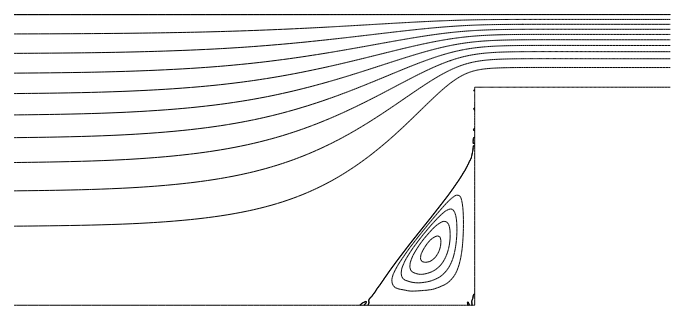

Fig. 8. Time-dependent inlet boundary conditions: transient streamlines, 4:1 contraction, $\left[\mathrm{CT} 3-\mathrm{LDB}, \delta_{\mathrm{MDC}}=1\right]$, Oldroyd-B, $W e=0.3$. 
a) $t=0.33$

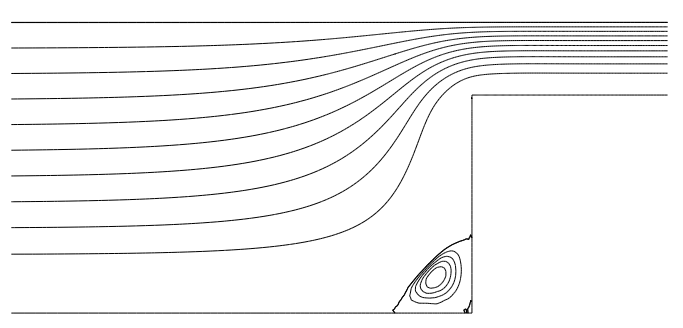

a) $t=0.63$

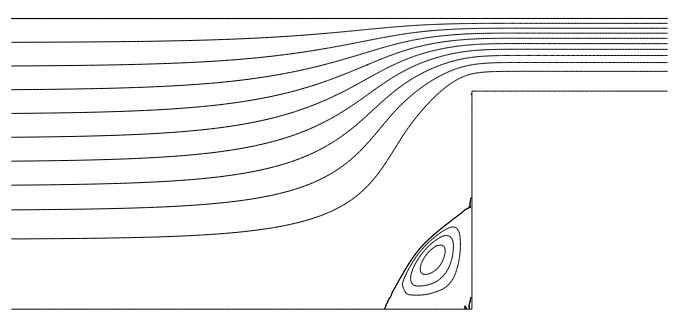

b) $t=1.23$

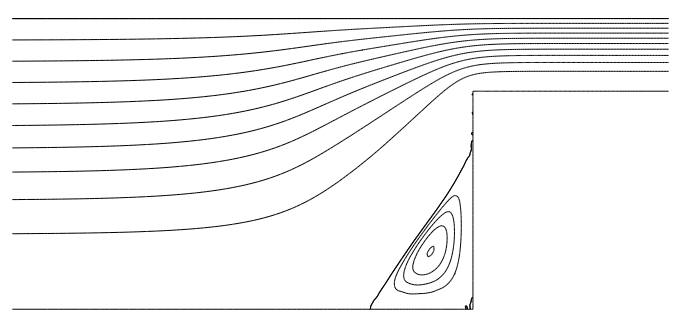

c) $t=1.82$

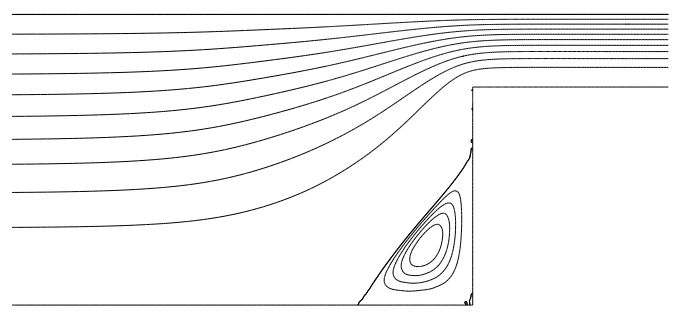

d) $t=3.10$

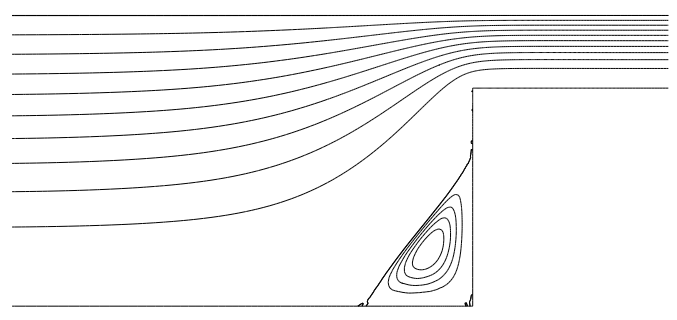

e) $t=3.49$ (Steady-state)

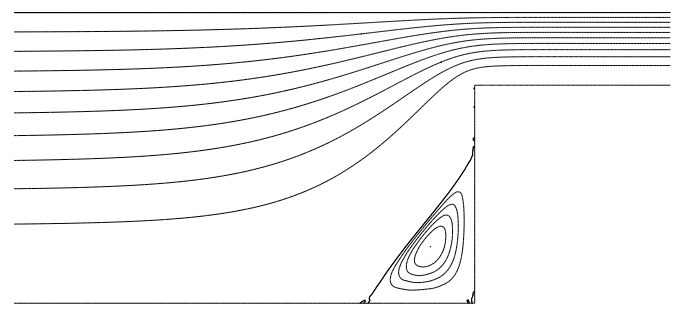

Fig. 9. Static inlet boundary conditions: transient streamlines, 4:1 contraction, $\left[\mathrm{CT} 3-\mathrm{LDB}, \delta_{\mathrm{MDC}}=1\right]$, Oldroyd-B, $W e=0.3$. 
a) $t=0.20$

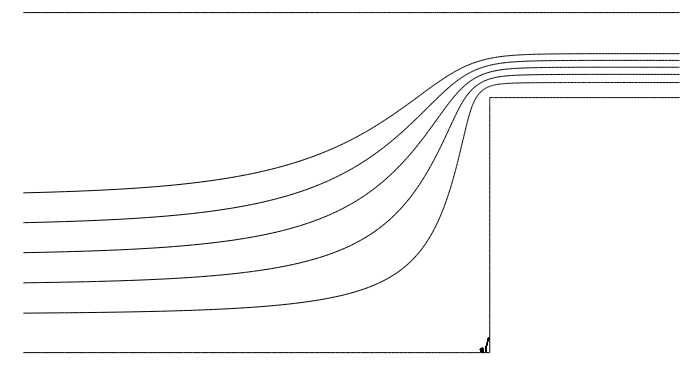

a) $t=0.53$

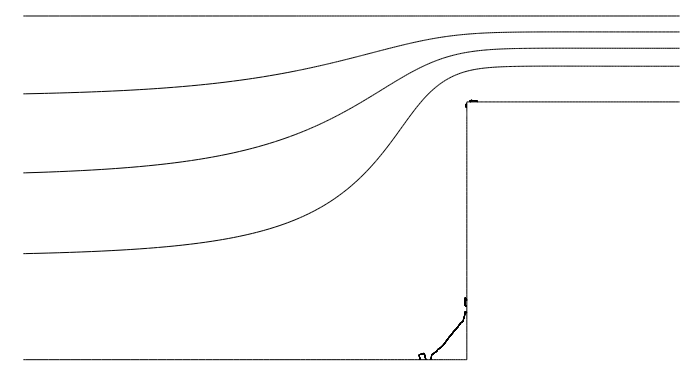

b) $t=0.69$

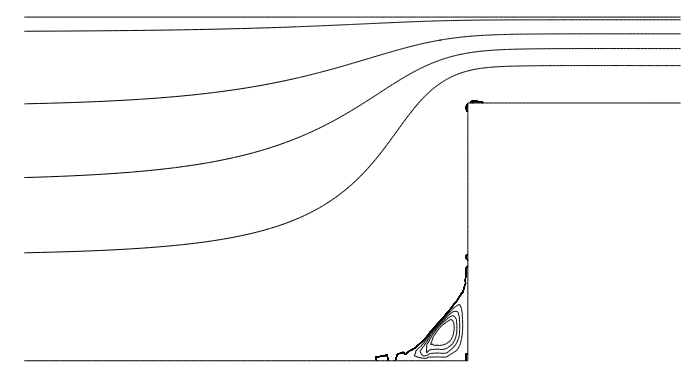

c) $t=1.40$

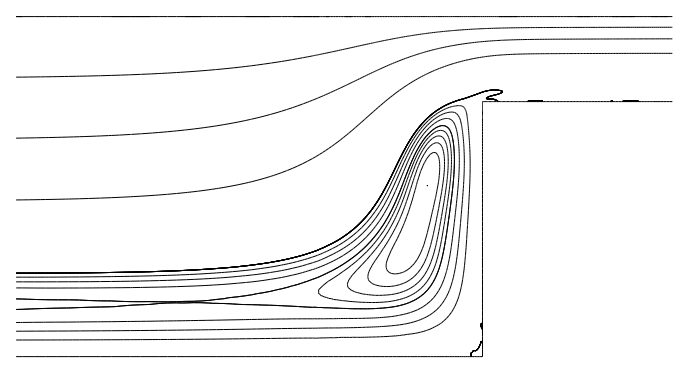

d) $t=2.20$

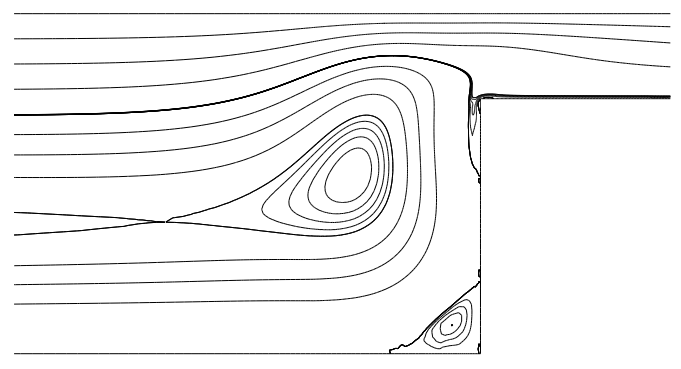

e) $t=2.90$

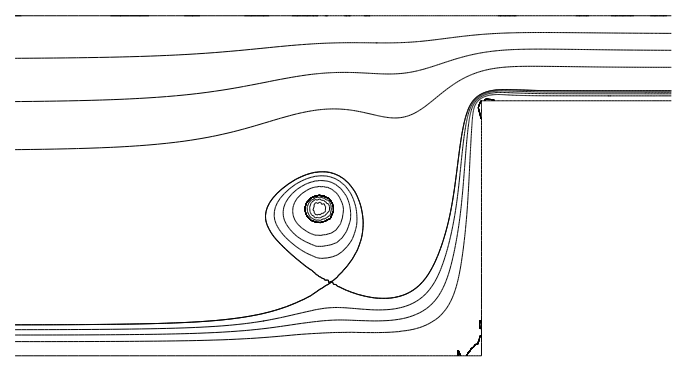

Fig. 10. Time-dependent inlet boundary conditions: transient streamlines, 4:1 contraction, [CT3-LDB, $\delta_{\mathrm{MDC}}=1$ ], Oldroyd-B, $W e=2$ (early evolution). 
a) $t=3.10$

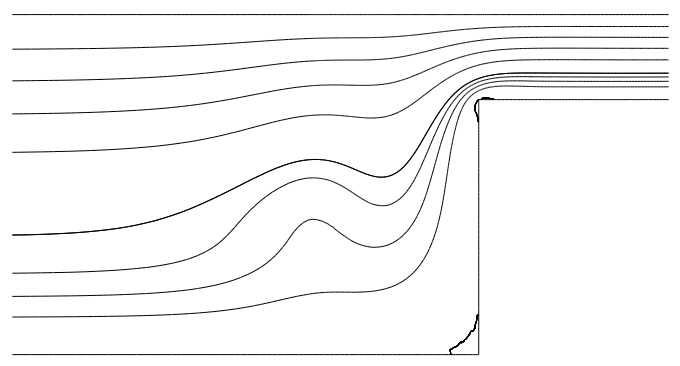

a) $t=3.71$

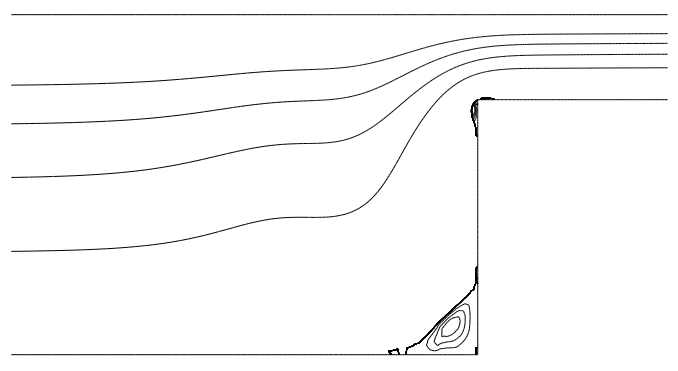

b) $t=5.00$

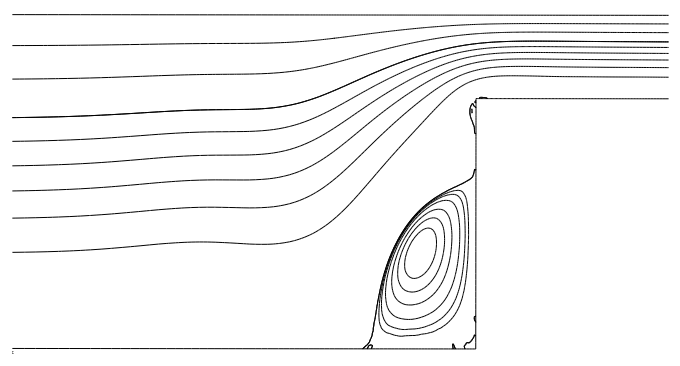

c) $t=10.0$

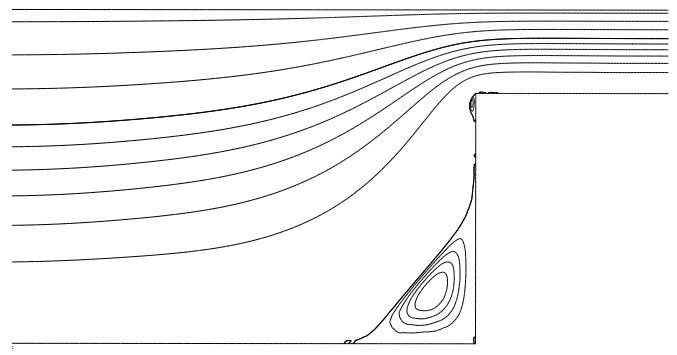

d) $t=20.0$

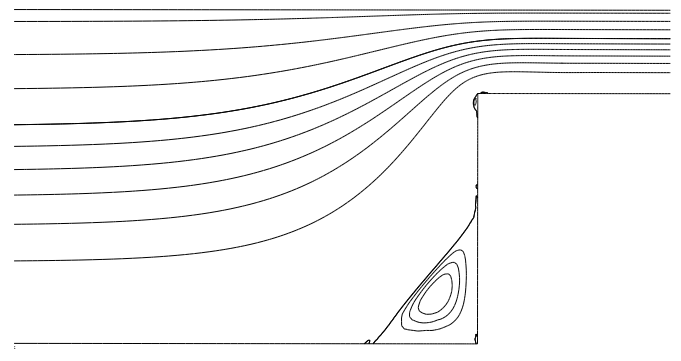

e) $t=59.7$ (Steady-state)

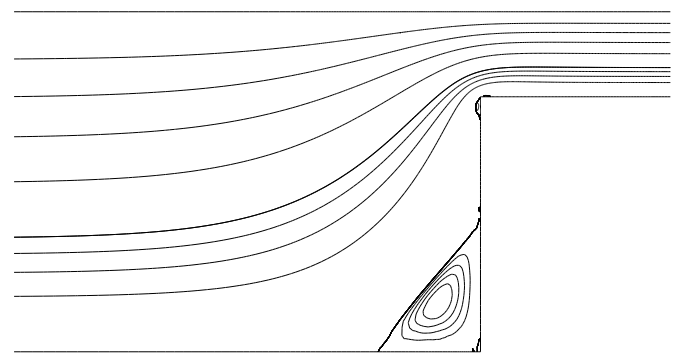

Fig. 11. Time-dependent inlet boundary conditions: transient streamlines, 4:1 contraction, [CT3-LDB, $\delta_{\mathrm{MDC}}=1$ ], Oldroyd-B, $W e=2$. 
a) $t=0.20$

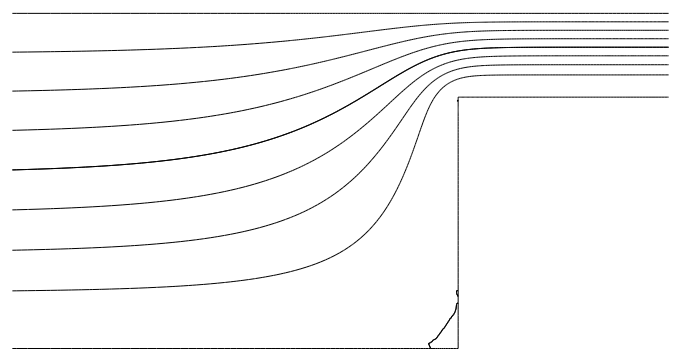

a) $t=0.53$

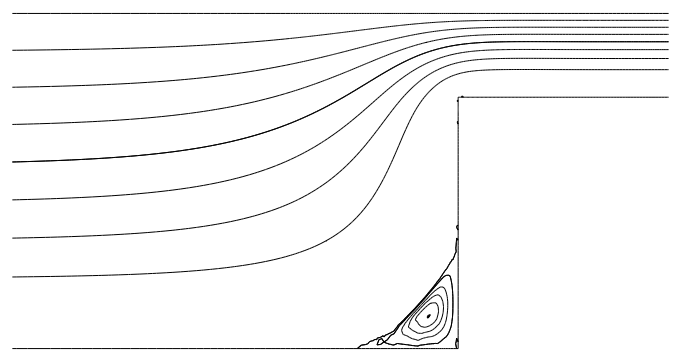

b) $t=0.69$

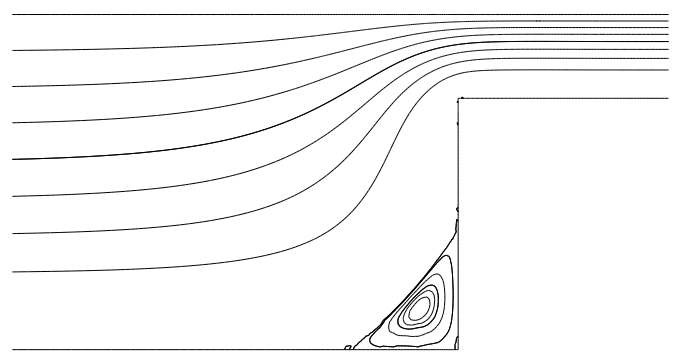

c) $t=1.40$

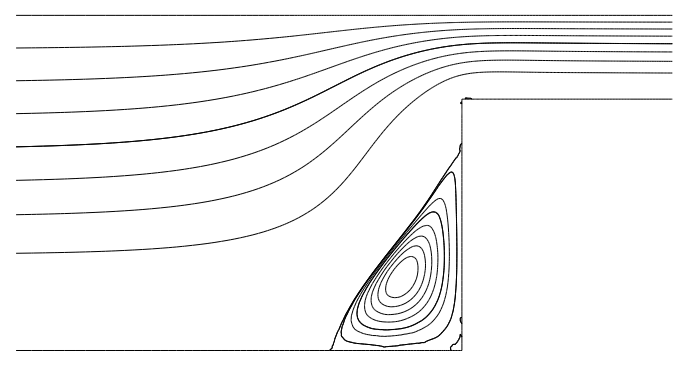

d) $t=2.20$

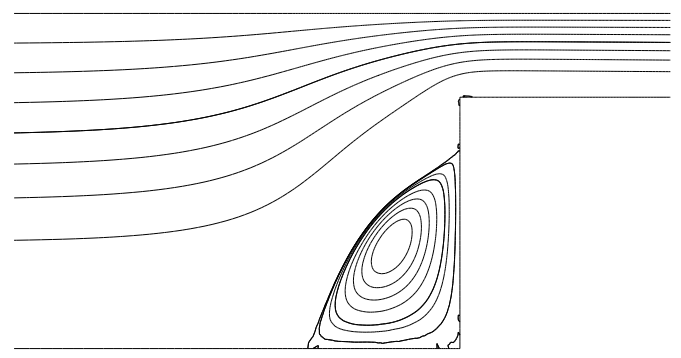

e) $t=2.90$

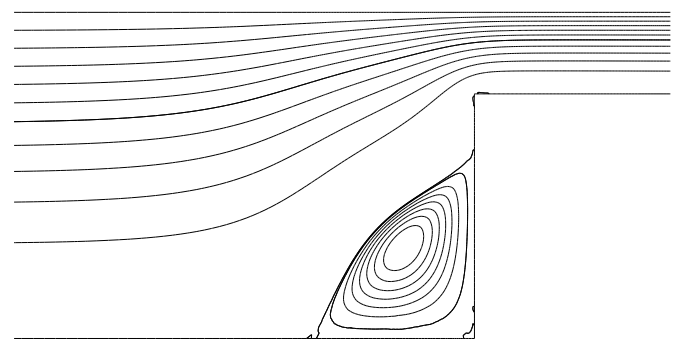

Fig. 12. Static inlet boundary conditions: transient streamlines, 4:1 contraction, $\left[\mathrm{CT} 3-\mathrm{LDB}, \delta_{\mathrm{MDC}}=1\right]$, Oldroyd-B, $W e=2$ (early evolution). 
a) $t=3.10$

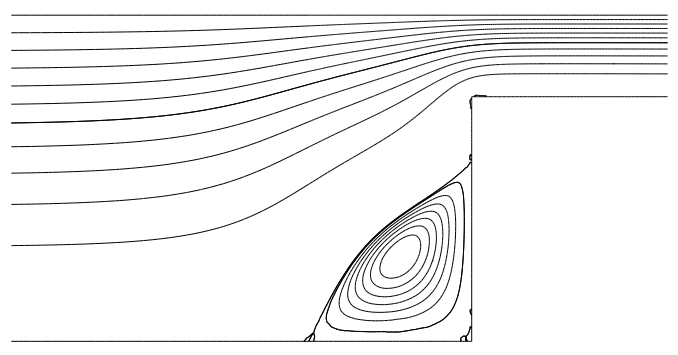

a) $t=3.71$

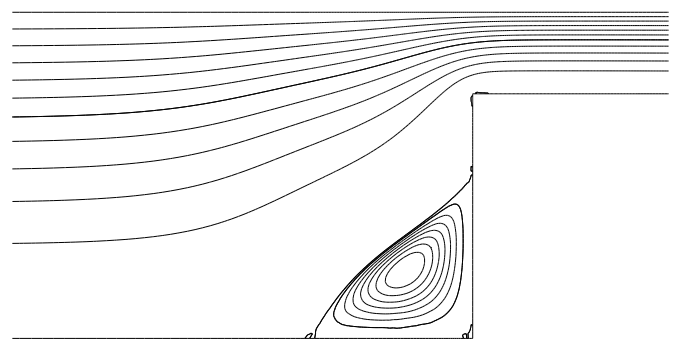

b) $t=5.00$

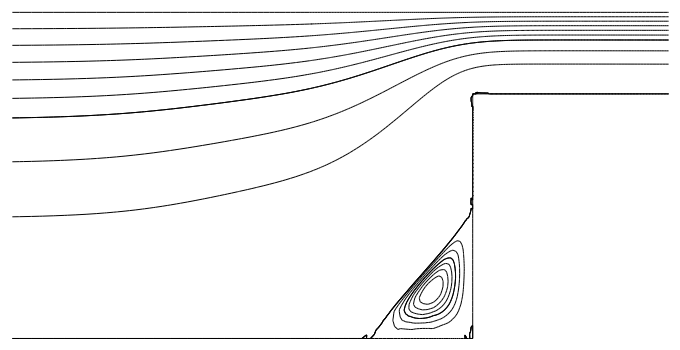

c) $t=10.0$

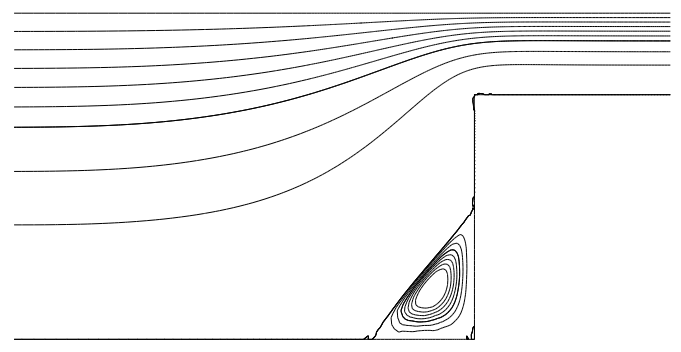

d) $t=20.0$

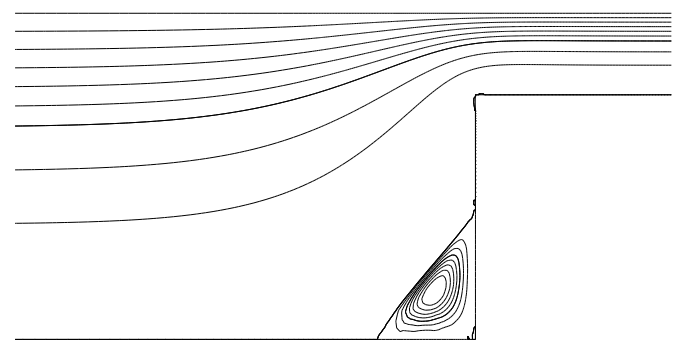

e) $t=34.8$ (Steady-state)

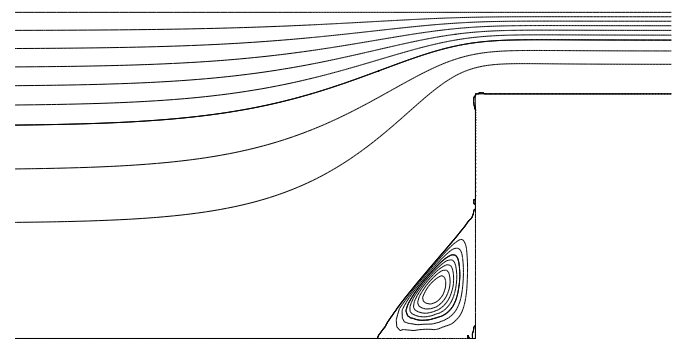

Fig. 13. Static inlet boundary conditions: transient streamlines, 4:1 contraction, $\left[\mathrm{CT} 3-\mathrm{LDB}, \delta_{\mathrm{MDC}}=1\right]$, Oldroyd-B, $W e=2$. 
a) $t=0.53$

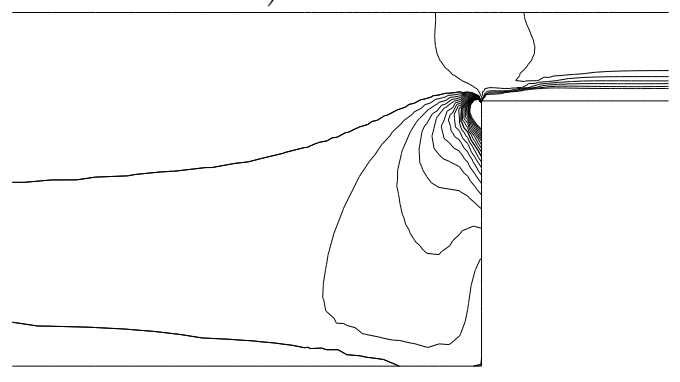

b) $t=0.69$

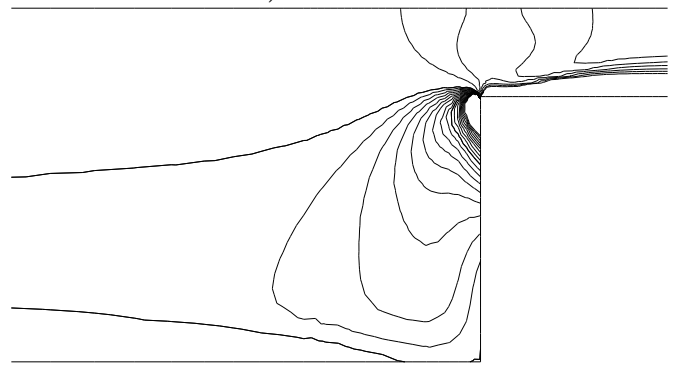

c) $t=1.40$

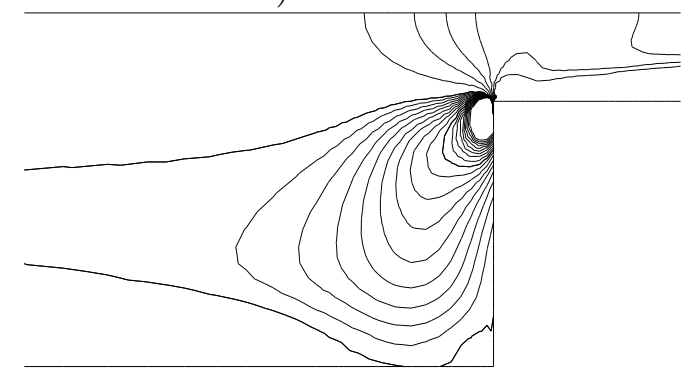

d) $t=2.20$

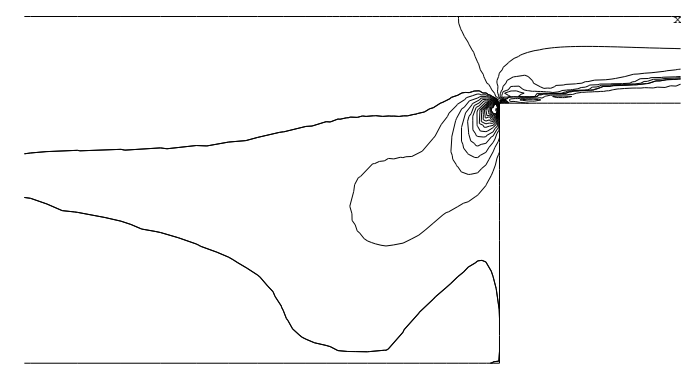

e) $t=2.90$

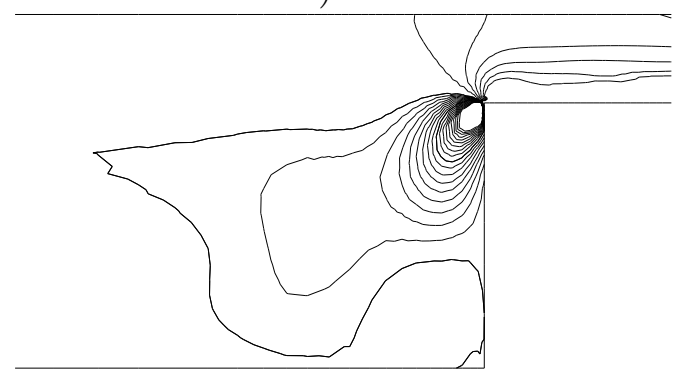

f) $t=3.10$

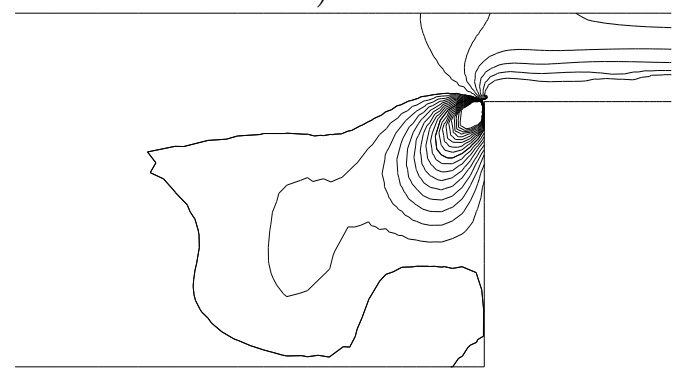

g) $t=3.71$

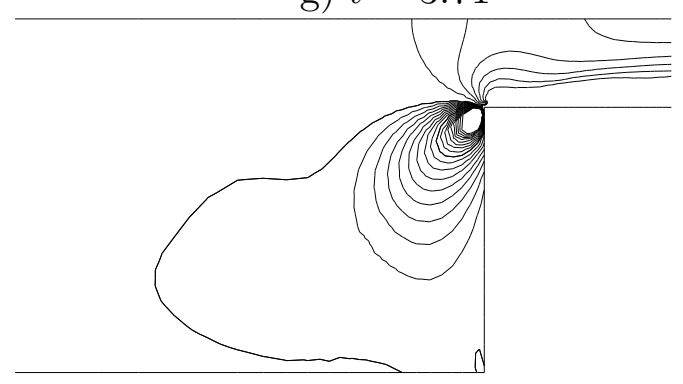

h) $t=59.7$ (steady-state)

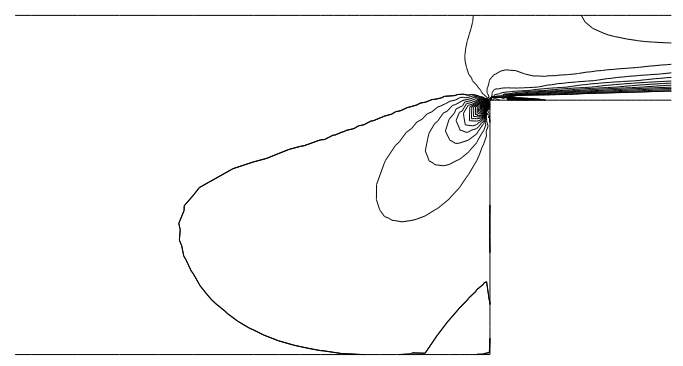

Fig. 14. Time-dependent inlet boundary conditions: transient $\tau_{x x}-\tau_{y y}$ fields, 4:1 contraction, [CT3-LDB, $\delta_{\mathrm{MDC}}=1$ ], Oldroyd-B, $W e=2$. 
a) $t=0.53$

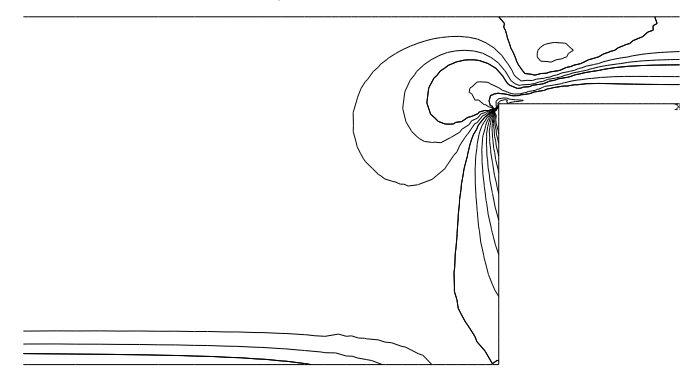

b) $t=0.69$

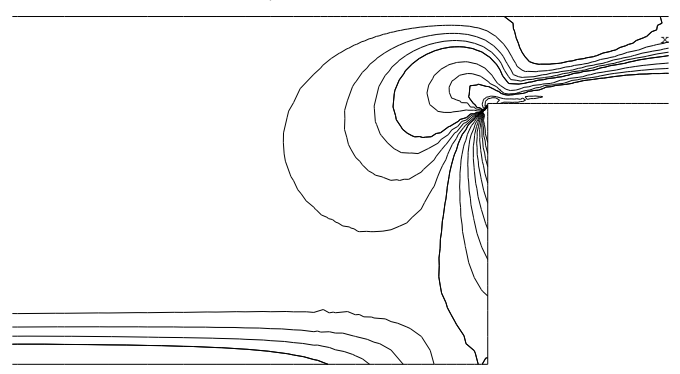

c) $t=1.40$

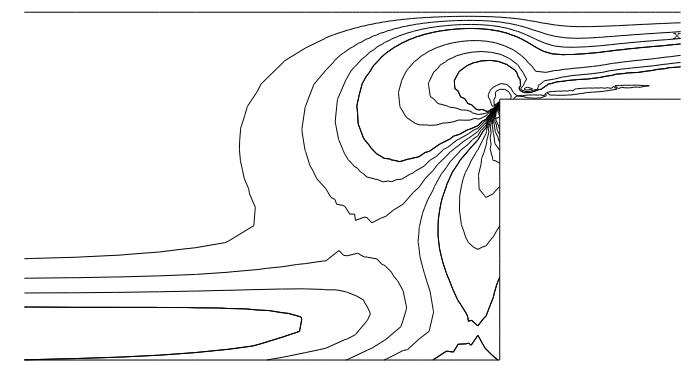

d) $t=2.20$

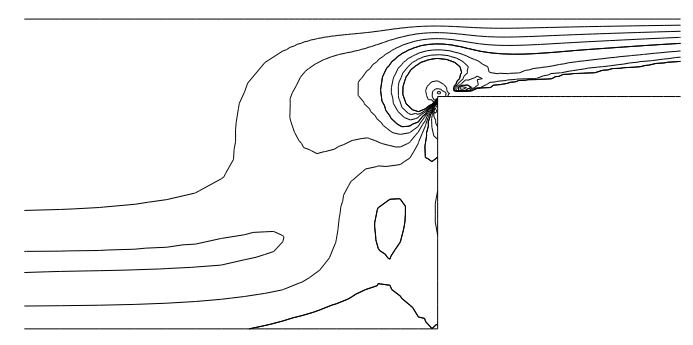

e) $t=2.90$

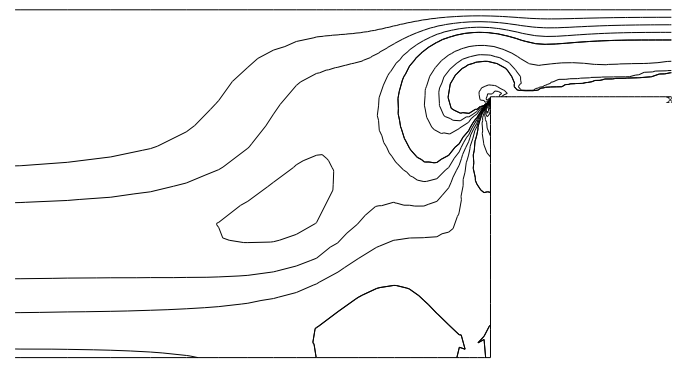

f) $t=3.10$

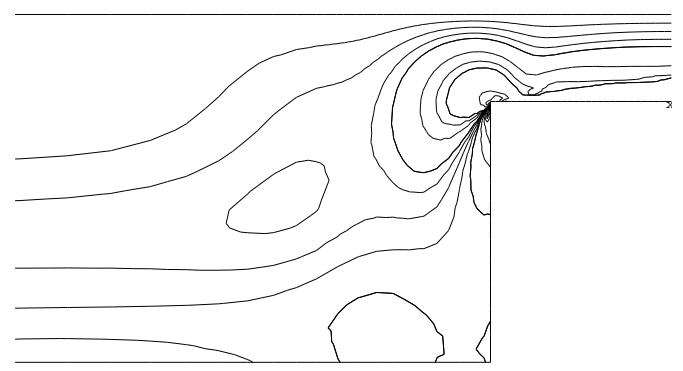

g) $t=3.71$

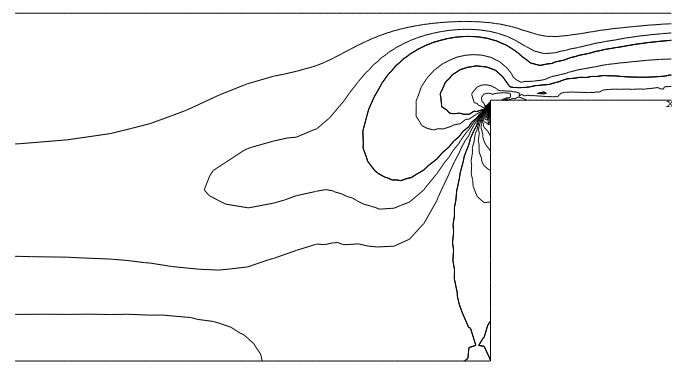

h) $t=59.7$ (steady-state)

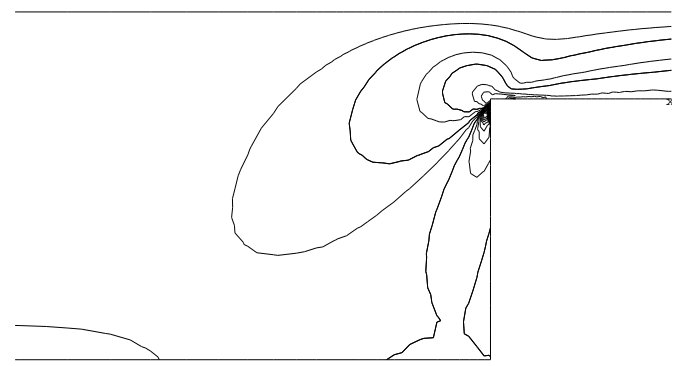

Fig. 15. Time-dependent inlet boundary conditions: transient $\tau_{x y}$ fields, $4: 1$ contraction, $\left[\mathrm{CT} 3-\mathrm{LDB}, \delta_{\mathrm{MDC}}=1\right]$, Oldroyd-B, $W e=2$. 

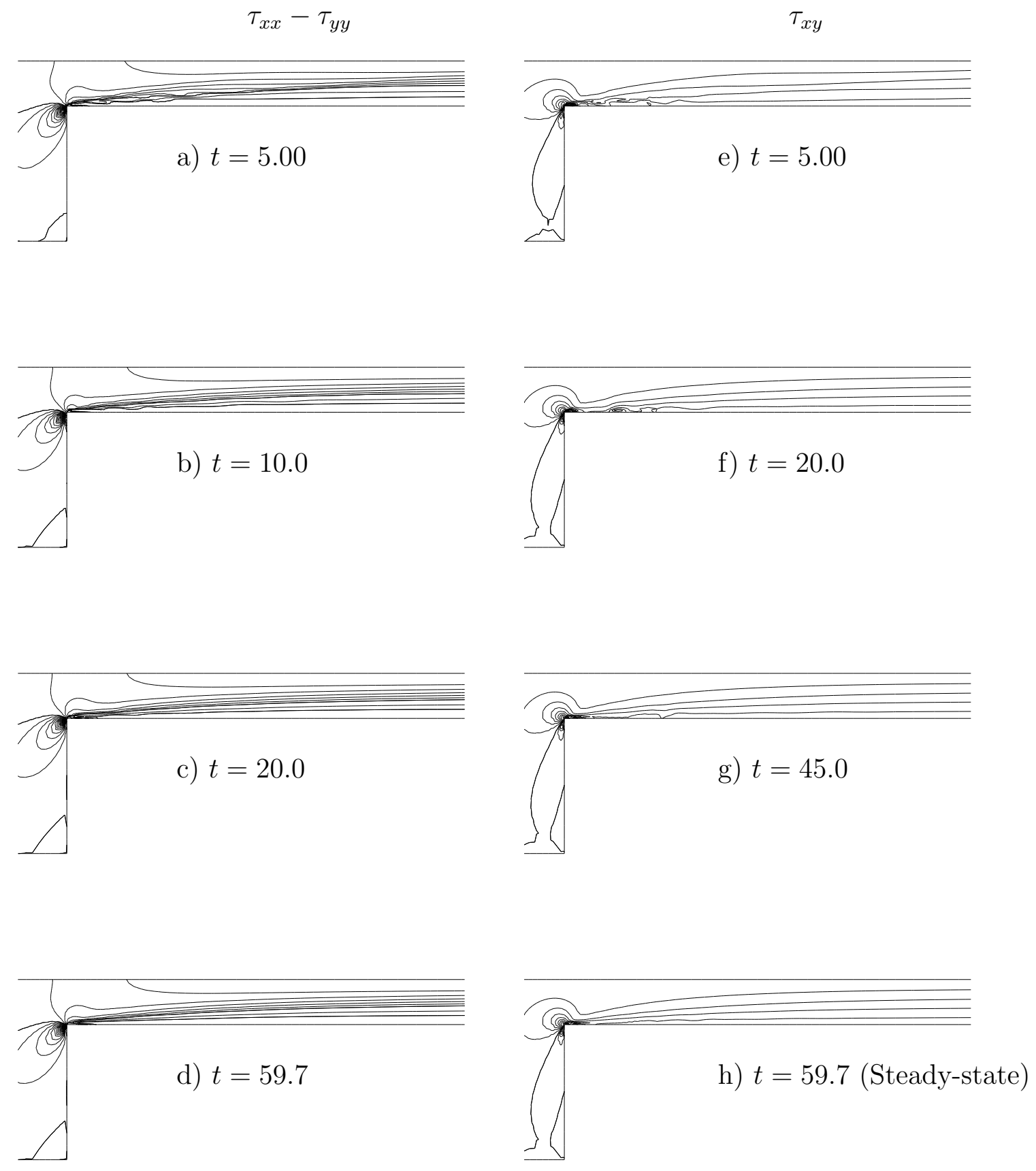

Fig. 16. Downstream-wall, transient stress boundary layers development: $\tau_{x x}-\tau_{y y}$ and $\tau_{x y}$ fields; 4:1 contraction, [CT3-LDB, $\delta_{\mathrm{MDC}}=1$ ], Oldroyd-B, $W e=2$. 